TRANSACTIONS OF THE

AMERICAN MATHEMATICAL SOCIETY

Volume 358, Number 11, November 2006, Pages 4765-4792

S 0002-9947(05)03792-X

Article electronically published on October 31, 2005

\title{
ON NEOCLASSICAL SCHOTTKY GROUPS
}

\author{
RUBÉN HIDALGO AND BERNARD MASKIT
}

\begin{abstract}
The goal of this paper is to describe a theoretical construction of an infinite collection of non-classical Schottky groups. We first show that there are infinitely many non-classical noded Schottky groups on the boundary of Schottky space, and we show that infinitely many of these are "sufficiently complicated". We then show that every Schottky group in an appropriately defined relative conical neighborhood of any sufficiently complicated noded Schottky group is necessarily non-classical. Finally, we construct two examples; the first is a noded Riemann surface of genus 3 that cannot be uniformized by any neoclassical Schottky group (i.e., classical noded Schottky group); the second is an explicit example of a sufficiently complicated noded Schottky group in genus 3 .
\end{abstract}

\section{INTRODUCTION}

It was shown by Marden 9] that there are Schottky groups that are not classical (that is, they cannot be defined by circles); see also [6]. An explicit family of examples of non-classical Schottky groups was constructed by Yamamoto [18. The main goal of this paper is to give a theoretical construction of infinitely many nonclassical Schottky groups. A geometrically finite Kleinian group on the boundary of Schottky space (i.e., a geometrically finite free Kleinian group that contains parabolic elements) is a noded Schottky group; the noded Schottky group is neoclassical if it can be defined by Euclidean circles. We show that, for each genus $\geq 2$, there are infinitely many topologically distinct noded Schottky groups, that only finitely many of these topologically distinct noded groups can be neoclassical, and that all but finitely many of these non-neoclassical noded Schottky groups are sufficiently complicated. That is, they are so far from being neoclassical that "nearby" Schottky groups are also not classical. It is an open question as to whether every closed Riemann surface can be uniformized by a classical Schottky group. We give a family of examples in genus 3 of noded Riemann surfaces that cannot be uniformized by neoclassical Schottky groups, thus answering in the negative the corresponding question for noded Riemann surfaces in the Deligne-Mumford compactification of moduli space. The basic ideas used in the proofs of the above facts stem from the following facts. Let $G$ be a noded Schottky group (i.e., a free, geometrically finite Kleinian group) with infinitely many components (of its set of discontinuity), all simply connected. Then, if $G$ is free on $p$ generators, there are $2 p$ almost disjoint simple closed curves on the Riemann sphere bounding a disconnected region, so

Received by the editors March 25, 2002 and, in revised form, July 21, 2004.

2000 Mathematics Subject Classification. Primary 30F10, 30F40.

This work was partially supported by Projects Fondecyt 1030252, 1030373, 7000715 and UTFSM 12.03.21. 
that there are generators of $G$ pairing these curves. Such curves are called defining loops for $G$. Since each component is simply connected, every defining loop must pass through some number of components; it must enter and leave each such component by passing through a parabolic fixed point. It is essentially immediate that if two distinct translates of some defining loop or loops both pass through two distinct parabolic fixed points, then they cannot both be circles. In particular, if two translates of some defining loop or loops both pass through some component, entering and leaving the component by the same parabolic fixed points, then they cannot both be circles. We will show below that if there are two distinct translates of some defining loop or loops which pass through two successive components, entering and leaving each through the same parabolic fixed points, then there are necessarily four points on at least one of them so that the imaginary part of the cross-ratio of these four points is uniformly bounded away from zero.

One can regard the noded Schottky groups as being on the boundary of the space of Schottky groups. Other boundaries of the algebraic topology space of algebraically marked Schottky groups have been studied by, among others, Gerritzen and Herrlich [2], Hidalgo [3] and Sato [16].

\section{Schottky Groups AND NODED Schottky Groups}

1.1. Kleinian groups. Throughout this paper, we will use the term Kleinian group to denote a discrete subgroup of $P S L(2, \mathbb{C})$, which we identify as both the group of orientation-preserving conformal homeomorphisms of the extended complex plane, $\widehat{\mathbb{C}}$, and as the group of orientation-preserving isometries of hyperbolic 3 -space.

Every Kleinian group $G$ decomposes $\widehat{\mathbb{C}}$ into two sets: the limit set $\Lambda=\Lambda(G)$, and its complement, the regular set (or discontinuity set) $\Omega=\Omega(G)$. The limit set is defined as the set of limit points of the set $\{g(x): g \in G\}$, where $x$ is any point in hyperbolic 3 -space.

It is well known that the limit set is either finite, consisting of at most two points, or is an uncountable perfect nowhere dense subset of $\widehat{\mathbb{C}}$, or is equal to $\widehat{\mathbb{C}}$. The Kleinian group is elementary if $\Lambda$ consists of at most two points. It is of the first kind if $\Lambda=\widehat{\mathbb{C}}$; it is of the second kind otherwise.

1.2. Definitions of Schottky groups. There are several equivalent definitions of a Schottky group; we start with the traditional one.

Definition 1. A Schottky group is a Kleinian group $G$ generated by transformations $a_{1}, \ldots, a_{p}$, where there are $2 p$ disjoint simple loops, $C_{1}, C_{1}^{\prime}, \ldots, C_{p}, C_{p}^{\prime}$, bounding a common domain $D$ in the extended complex plane $\widehat{\mathbb{C}}$, where $a_{i}\left(C_{i}\right)=C_{i}^{\prime}$, and $a_{i}(D) \cap D=\emptyset, i=1, \ldots, p$.

In the above, the set of loops $C_{1}, \ldots, C_{p}^{\prime}$ are called defining loops for the Schottky generators $a_{1}, \ldots, a_{p}$. If we do not need the generators, we will sometimes simply refer to the set of loops as defining loops. The value $p$ is called the genus of the Schottky group, and we sometimes refer to $G$ as a Schottky group of genus $p$. Let $\Omega=\Omega(G)$ be the set of discontinuity of $G$. It is well known that $\Omega=\Omega(G)$ is connected and dense in $\widehat{\mathbb{C}}$, and that $S=\Omega / G$ is a closed Riemann surface of genus $p$. Also, if we let $V_{i}$ be the projection of $C_{i}$ to $S$, then $V_{1}, \ldots, V_{p}$ is a set of simple disjoint homologically independent loops. We also call $V_{1}, \ldots, V_{p}$ a set of defining 
loops for $G$. It is also well known that a Schottky group is finitely generated, free and purely loxodromic. It was shown in [10] that these properties characterize these groups within the class of Kleinian groups of the second kind.

Definition 2. A Schottky group is a finitely generated, free Kleinian group of the second kind, in which every non-trivial element is loxodromic (including hyperbolic). It is well known that every Schottky group is geometrically finite. A geometrically finite Kleinian group without parabolics is either co-compact or of the second kind. Since a free group cannot be the fundamental group of a closed 3 -manifold, we can restate this second definition as follows.

Definition $2^{\prime}$. A Schottky group is a free geometrically finite Kleinian group without parabolics.

The following equivalent definition is essentially Koebe's retrosection theorem; a modern proof can be found in [13, p. 312].

Definition 3. Let $S$ be a closed Riemann surface of genus $p$, and let $V_{1}, \ldots, V_{p}$ be $p$ homologically independent disjoint simple closed loops on $S$. Each of these loops is homologically non-trivial, hence also homotopically non-trivial. Then the highest regular covering of $S$ for which $V_{1}, \ldots, V_{p}$ all lift to loops is conformally an open subset of $\widehat{\mathbb{C}}$. The group of deck transformations for this covering, as a group of conformal maps of a plane domain, is a Schottky group of genus $p$.

We include the fourth definition, which we will not use, for the sake of completeness; its equivalence With Definition 2 is well known.

Definition 4. Let $M$ be a handlebody of genus $p$ endowed with a geometrically finite complete hyperbolic structure, where the injectivity radius is bounded from below by a positive constant. Then the universal covering group of $M$, acting on hyperbolic 3 -space $\mathbb{H}^{3}$, is a Schottky group of genus $p$.

1.3. Definitions of noded Schottky groups. It is clear that if we permit the defining loops of a Schottky group to touch without crossing at a finite number of points, we will still generate a free Kleinian group of the second kind. However, in order to guarantee that the common outside of all the curves is a fundamental domain, we need to ensure that these new points of contact are parabolic fixed points (see [14]).

Definition 5. A noded Schottky group is a Kleinian group $\widehat{G}$ generated by Möbius transformations $\widehat{a}_{1}, \ldots, \widehat{a}_{p}$, where there are $2 p$ simple loops, $\widehat{C}_{1}, \widehat{C}_{1}^{\prime}, \ldots, \widehat{C}_{p}, \widehat{C}_{p}^{\prime}$, satisfying the following conditions:

(i) Any two of these loops intersect in at most finitely many points;

(ii) there are pairwise disjoint open discs, $\widehat{D}_{1}, \widehat{D}_{1}^{\prime}, \ldots, \widehat{D}_{p}, \widehat{D}_{p}^{\prime}$, so that the boundary of $\widehat{D}_{i}$ is $\widehat{C}_{i}$ and the boundary of $\widehat{D}_{i}^{\prime}$ is $\widehat{C}_{i}^{\prime}$;

(iii) $\widehat{a}_{i}\left(\widehat{C}_{i}\right)=\widehat{C}_{i}^{\prime}$, and $\widehat{a}_{i}\left(\widehat{D}_{i}\right) \cap \widehat{D}_{i}^{\prime}=\emptyset, i=1, \ldots, p$;

(iv) each point of intersection of any two defining loops is a fixed point of a parabolic transformation in $\widehat{G}$.

As above, the loops $\widehat{C}_{i}, \widehat{C}_{i}^{\prime}$ are called defining loops for the noded Schottky group, and the generators, $\widehat{a}_{1}, \ldots, \widehat{a}_{p}$ are called noded Schottky generators. The value $p$ is called the genus of $G$, and we also say that $G$ is a noded Schottky group of 
genus $p$. It was shown in [14 that every noded Schottky group is geometrically finite; that every parabolic element of $\widehat{G}$ is a conjugate of a power of one of the transformations fixing a common point of two of the defining loops, and, if we let $\widehat{D}$ denote the complement of the union of the closures of $\widehat{D}_{1}, \widehat{D}_{1}^{\prime}, \ldots, \widehat{D}_{p}, \widehat{D}_{p}^{\prime}$, that $\widehat{D}$ is a fundamental domain for $\widehat{G}$. The fact that a geometrically finite free Kleinian group of the second kind is a noded Schottky group was proved in [11. The fact that every geometrically finite free Kleinian group is of the second kind was proved in 3 .

Definition 6. A noded Schottky group is a geometrically finite free Kleinian group.

Since we will not need it, we will not state the (complicated and difficult) analog of Definition 3 for noded Schottky groups. However, we do note that the analog of Definition 4 is quite simple.

Definition 7. Let $M$ be a handlebody of genus $p$ with a geometrically finite hyperbolic structure. Then the universal covering group of $M$, acting on $\mathbb{H}^{3}$, is a noded Schottky group of genus $p$.

In the definitions above, every Schottky group is also a noded Schottky group. A proper noded Schottky group is one that necessarily contains parabolic elements.

1.4. Generators vs. groups. Throughout this paper, we will deal with Schottky groups and noded Schottky groups, sometimes marked topologically by a set of defining loops, including a set of generators, sometimes only marked by a set of generators. In any case, we will usually regard these generators as being given up to conjugation in $\operatorname{PSL}(2, \mathbb{C})$. Chuckrow's theorem [1] asserts that every set of free generators for a Schottky group is a set of Schottky generators; that is, if $a_{1}, \ldots, a_{p}$ is any set of free generators for a Schottky group, then there are defining loops $C_{1}, \ldots, C_{p}^{\prime}$ for these generators as in Definition 1. One expects however that the corresponding statement is not true for noded Schottky groups (this will be explained below). Let $\widehat{G}$ be a noded Schottky group of genus $p$. If we adjoin the parabolic fixed points of $\widehat{G}$ to $\Omega(\widehat{G})$, with the appropriate cusped topology (see 4 or $[8)$, we obtain the set $\Omega^{+}(\widehat{G})$, called the noded set of discontinuity of $\widehat{G}$. One then easily sees that $S^{+}=\Omega^{+} / \widehat{G}$ is a noded closed Riemann surface of genus $p$. It was observed in 3 that, given any noded closed Riemann surface $S^{+}$of genus $p$, there is a noded Schottky group $\widehat{G}$ (necessarily of genus $p$ ) so that $S^{+}=\Omega^{+} / \widehat{G}$. It follows that every point of the Deligne-Mumford compactification of the moduli space of genus $p$ can be realized by a noded Schottky group of genus $p$. It was shown in [1] that there are $p$ (smooth) loops on $S^{+}, \widetilde{W}_{1}, \ldots, \widetilde{W}_{p}$, which lift to loops on $\Omega^{+}$, and which, except that they may pass through the nodes, are simple and disjoint; at the nodes, they touch but do not cross (that is, if two of these loops pass through a node, then they are tangent to each other at each side of the node; since the two sides of the node are consistently oriented, and the curves are smooth, one can follow them across the node to see if they cross or not). By using Chuckrow's theorem, one easily sees that the result in [11 in fact yields the following. Let $\widehat{a}_{1}, \ldots, \widehat{a}_{p}$ be any set of free generators of $\widehat{G}$. Then we can choose the $p$ loops, $\widetilde{W}_{1}, \ldots, \widetilde{W}_{p}$, on $S^{+}$, so that if we lift the complement of these loops (including the appropriate nodes) to $\Omega^{+}(\widehat{G})$, then we obtain a fundamental domain for $\widehat{G}$, bounded by $2 p$ not necessarily simple loops. These $2 p$ loops come in pairs, 
where each pair, say $\widehat{C}_{i}$ and $\widehat{C}_{i}^{\prime}$, are both lifts of the same $\widetilde{W}_{i}$; further, for each $i=1, \ldots, p$, this pairing is explicitly accomplished by the fact that $\widehat{a}_{i}\left(\widehat{C}_{i}\right)=\widehat{C}_{i}^{\prime}$. As suggested above, while these loops, $\widehat{C}_{1}, \widehat{C}_{1}^{\prime}, \ldots, \widehat{C}_{p}, \widehat{C}_{p}^{\prime}$, are almost disjoint (that is, they meet without crossing at some number of parabolic fixed points), they need not be simple - that is, some of them might pass through the same parabolic fixed point more than once. It might even be that if we start with a given set of free generators for a noded Schottky group, then there is no choice of a set of simple almost disjoint defining loops for these generators.

1.5. Rigid groups. In general, a rigid Kleinian group is one that admits no (quasiconformal) deformations. It is well known that a geometrically finite Kleinian group of the second kind $G$ is rigid if and only if $\Omega(G) / G$ is a finite union of thricepunctured spheres. We will need the fact that if $\widehat{G}$ is a rigid noded Schottky group, then every component of $\widehat{G}$ (i.e., connected component of $\Omega(\widehat{G})$ ) is a Euclidean disc, and, if one factors this Euclidean disc by its stabilizer, one obtains a thricepunctured sphere. The Fuchsian group stabilizing this Euclidean disc is a conjugate of the classical Fuchsian $(\infty, \infty, \infty)$-triangle group.

1.6. Classical and neoclassical groups. If $G$ is a Schottky group, given by a set of defining loops all of which are Euclidean circles, then $G$ is classical, and the corresponding generators form a classical generating set. If $\widehat{G}$ is a noded Schottky group given by a set of defining loops all of which are Euclidean circles, then $\widehat{G}$ is a neoclassical Schottky group, and the corresponding set of generators is a neoclassical set of generators.

There are Schottky groups for which every set of free generators is classical. For example, every finitely generated purely hyperbolic Fuchsian group representing a closed surface with holes, is, as a Kleinian group, a Schottky group. In the special case that the Fuchsian group is a two generator group representing a torus with one hole, this Schottky group is classical on every set of generators.

On the other hand, regardless of whether $G$ is classical or not, if $a_{1}, \ldots, a_{p}$ is a set of free generators for $G$, and no set of defining loops with these generators consists only of Euclidean circles, then $a_{1}, \ldots, a_{p}$ forms a non-classical set of generators. For example, if the Fuchsian group represents a sphere with three holes, then it has infinitely many sets of generators that are classical and infinitely many sets of generators that are non-classical. One expects that in general, classical Schottky groups have many sets of generators that are non-classical.

For noded Schottky groups, if $\widehat{a}_{1}, \ldots, \widehat{a}_{p}$ is a set of free generators for the noded Schottky group $\widehat{G}$, and every set of defining loops for these generators contains at least one loop that is not a Euclidean circle, then $\widehat{a}_{1}, \ldots, \widehat{a}_{p}$ is a non-neoclassical set of generators. As with Schottky groups, one expects that in general a noded Schottky group will have many sets of generators that are not neoclassical. One also expects that in general a neoclassical Schottky group will have many sets of generators that are not neoclassical.

The Schottky group $G$ is classical if it has at least one set of classical generators; it is non-classical otherwise. Similarly, the noded Schottky group $\widehat{G}$ is neoclassical if it has at least one set of neoclassical generators; it is non-neoclassical otherwise. 


\section{The Boundary of Schottky Space}

There are several natural spaces of Schottky groups (see [15]), and one can regard some of our constructions here as that of putting the appropriately marked noded Schottky groups on the boundaries of these spaces. These ideas will be pursued elsewhere. We will need the usual space of deformations of a Schottky group of genus $p$, denoted by $\mathcal{S}_{a l g}$, which we will usually think of as a subset of the representation space of the free group of rank $p$ in $P S L(2, \mathbb{C})$, modulo conjugation.

2.1. The infinite shoebox construction. Let $\widehat{C}_{1}, \ldots, \widehat{C}_{p}^{\prime}$, with generators $\widehat{a}_{1}, \ldots$, $\widehat{a}_{p}$, be a set of defining loops for a noded Schottky group $\widehat{G}$, and let $\widehat{p}_{1}, \ldots, \widehat{p}_{q}$ be a maximal set of primitive parabolic elements of $\widehat{G}$ generating non-conjugate cyclic subgroups. For each $i=1, \ldots, q$, choose a particular Möbius transformation $h_{i}$ conjugating $\widehat{p}_{i}$ to the transformation $P(z)=z+1$. Consider the renormalized group $h_{i} \widehat{G} h_{i}^{-1}$. For this group, there is a number $\alpha_{0}>1$ so that the set $\left\{|\Im(z)| \geq \alpha_{0}\right\}$ is precisely invariant under $\operatorname{Stab}(\infty) \subset h_{i} \widehat{G} h_{i}^{-1}$. Regard $\mathbb{H}^{3}$ as being the set $\{(z, t): z \in \mathbb{C}, t>0 \in \mathbb{R}\}$. We likewise identify $\mathbb{C}$ with the boundary of $\mathbb{H}^{3}$, except for the point at infinity; that is, we identify $\mathbb{C}$ with $\{(z, t): t=0\}$.

In this normalization, for each parameter $\alpha$, with $\alpha>\alpha_{0}$, we define the infinite shoebox to be the set $B_{0, \alpha}=\{(z, t):|\Im(z)| \leq \alpha, t \leq \alpha\}$. Since $\alpha_{0}>1$, we easily observe that for every $\alpha>\alpha_{0}$, the complement of $B_{0, \alpha}$ in $\mathbb{H}^{3} \cup \mathbb{C}$ is precisely invariant under $\operatorname{Stab}(\infty) \subset h_{i} \widehat{G} h_{i}^{-1}$, where we are now regarding Möbius transformations as hyperbolic isometries, which act on the closure of $\mathbb{H}^{3}$. Then for $\widehat{G}$, the infinite shoebox with parameter $\alpha$ at $z_{i}$, the fixed point of $\widehat{p}_{i}$, is $B_{i, \alpha}=h_{i}^{-1}\left(B_{0, \alpha}\right)$, and, if $\widehat{p}$ is any parabolic element of $\widehat{G}$, conjugate to some power of $\widehat{p}_{i}$, then the corresponding infinite shoebox at $x$, the fixed point of $\widehat{p}$, is given by $b\left(B_{i, \alpha}\right)$, where $\widehat{p}=b \widehat{p}_{i} b^{-1}$.

It was observed in [1] that, for each fixed $\alpha>\alpha_{0}, \widehat{G}$ acts as a group of conformal homeomorphisms on the expanded regular set $B^{\alpha}=\bigcap \widehat{g}\left(B_{i, \alpha}\right)$, where the intersection is taken over all $\widehat{g} \in \widehat{G}$ and all $i=1, \ldots, q$. Further, $\widehat{G}$ acts as a Schottky group (in the sense of Definition 3) on the boundary of $B^{\alpha}$. Observe that each parabolic $\widehat{g} \in \widehat{G}$ appears to have two fixed points on the boundary of $B^{\alpha}$; that is, $\widehat{g}$, as it acts on the boundary of $B^{\alpha}$, appears to be loxodromic. We define the flat part of $B^{\alpha}$ to be the intersection of $B^{\alpha}$ with the extended complex plane. The complement of the flat part (on the boundary of $B^{\alpha}$ ) is the disjoint union of 3-sided boxes, where each box has two vertical sides (translates of the sets $\{\Im(z)= \pm \alpha, 0<t<\alpha\}$ ) and one horoball side (a translate of the set $\{|\Im(z)| \leq \alpha, t=\alpha\}$ ).

We will need a particular class of relatively compact subsets of $B^{\alpha}$. For each $i=$ $1, \ldots, q$ and each $n=1,2, \ldots$, we define $B_{i, \alpha, n}$ to be $h_{i}^{-1}\left(B_{0, \alpha} \cap\{|\Re(z)| \leq n\}\right)$. Then we define $B^{\alpha, n}=\bigcap \widehat{g}\left(B_{i, \alpha, n}\right)$, and the truncated flat part of $B^{\alpha, n}$ as the intersection of $B^{\alpha, n}$ with the extended complex plane. The boundary of the truncated flat part near a parabolic fixed point, renormalized so as to lie at $\infty$, is a Euclidean rectangle.

We now renormalize $\widehat{G}$ so that $\infty$ is an interior point of $\Omega(\widehat{G})$. Then, for each $\alpha>\alpha_{0}$, there is a conformal map $f^{\alpha}$, mapping the boundary of $B^{\alpha}$ to $\widehat{\mathbb{C}}$, and conjugating $\widehat{G}$ onto a Schottky group $G^{\alpha}$, where $f^{\alpha}$ is defined by the requirement that, near $\infty, f^{\alpha}(z)=z+O\left(|z|^{-1}\right)$. We remark that the group $G^{\alpha}$ depends on the choice of the transformations $h_{1}, \ldots, h_{q}$ as well as on the choice of $\alpha$.

It was shown in 11 that, with the above normalization, $f^{\alpha} \rightarrow I$, where $I$ denotes the identity, uniformly on compact subsets of $\Omega(\widehat{G})$, and that, for each fixed $\widehat{g} \in \widehat{G}$, 
$f^{\alpha} \widehat{g}\left(f^{\alpha}\right)^{-1} \rightarrow \widehat{g}$, as $\alpha \rightarrow \infty$. In particular, if we fix $\alpha_{0}$, and fix $n$, then $f^{\alpha} \rightarrow I$ uniformly on compact subsets of the truncated flat part of $B^{\alpha_{0}, n}$.

The boundary of $B^{\alpha_{0}, n}$ consists of a disjoint union of quadrilaterals with circular sides. After renormalization, the part of the boundary of $B^{\alpha_{0}, n}$ corresponding to $\left\{|\Im(z)|=\alpha_{0}\right\}$ is the horizontal part of the boundary, while the part of the boundary corresponding to $\{|\Re(z)|=n\}$ is the vertical part of the boundary.

2.2. Vertical projection. We fix a choice of conjugating maps, $h_{i}, i=1, \ldots, q$, and we fix a choice of the parameter $\alpha>\alpha_{0}$.

We need to deform all the $\widehat{C}_{i}$ and $\widehat{C}_{i}^{\prime}$, within $\Omega^{+}(\widehat{G})$ to an equivalent defining set of loops, with the same generators, so that, after appropriate renormalization, each connected component of each of the deformed loops appears, in each component of the complement of the flat part of $B^{\alpha}$, as a pair of half-infinite Euclidean vertical lines, one in $\{\Im(z) \geq \alpha\}$, the other in $\{\Im(z) \leq-\alpha\}$, both with the same real part. This deformation is accomplished in several steps. We start by deforming the $\widehat{C}_{i}$ and $\widehat{C}_{i}^{\prime}$ so that they all meet the lines $\left\{\Im z=\alpha_{0}\right\}$ and $\left\{\Im z=-\alpha_{0}\right\}$ transversely in at most finitely many points, and that these points of intersection are all distinct.

We first observe that we can easily deform all the $\widehat{C}_{i}$ and $\widehat{C}_{i}^{\prime}$ so that each of them intersects both $\left\{\Im(z)>\alpha_{0}\right\}$ and $\left\{\Im(z)<-\alpha_{0}\right\}$ in at most one arc having one of its endpoints at the point at infinity. To accomplish this, we remark that we have required that each $C_{i}$ and each $C_{i}^{\prime}$ be simple, and so can pass at most once through $\infty$. Then, if there is another arc of some $C_{i}$ intersecting for example $\{\Im(z)>\alpha\}$, this arc starts and ends at two points on $\left\{\Im(z)=\alpha_{0}\right\}$, and the distance between these points is less than 1 . There are at most a finite number of other such arcs of the $C_{i}$ or $C_{i}^{\prime}$ lying between these two points of intersection, and there is one whose points of intersection on this line are closest. Since there is some $\epsilon>0$ so that the set $\left\{\Im(z)>\alpha_{0}-\epsilon\right\}$ is precisely invariant under $\operatorname{Stab}(\infty)$, we can easily pull this arc, and its appropriate image, down below the line $\left\{\Im(z)=\alpha_{0}\right\}$ while maintaining all the requisite properties for these loops. After a finite number of iterations of this step, we will have accomplished that each $\widehat{C}_{i}$ and each $\widehat{C}_{i}^{\prime}$ transversally intersects each of the lines $\left\{\Im z=\alpha_{0}\right\}$ and $\left\{\Im z=-\alpha_{0}\right\}$ at most once.

We now have some number of points of intersection of the loops $C_{i}$ and $C_{i}^{\prime}$ with the line $\left\{\Im(z)=\alpha_{0}\right\}$; call these $x_{1}, \ldots, x_{m}$, where $\Re\left(x_{1}\right)<\cdots<\Re\left(x_{m}\right)$. There are likewise $m$ points of intersection of these loops with the line $\left\{\Im(z)=-\alpha_{0}\right\}$; call these points of intersection $y_{1}, \ldots, y_{m}$, where $\Re\left(y_{i}\right)<\cdots<\Re\left(y_{m}\right)$.

Our next observation is that $\Re\left(x_{m}\right) \leq \Re\left(x_{1}\right)+1$. The loops $\widehat{C}_{k}$ and $\widehat{C}_{k}^{\prime}$ all lie on the boundary of the fundamental domain $\widehat{D}$. So the $\operatorname{arcs}$ of the line $\left\{\Im(z)=\alpha_{0}\right\}$ lying between these points of intersection are alternately in $\widehat{D}$ and in its complement. Since the transformation $P: z \rightarrow z+1$ must map $\widehat{D}$ into its complement, the region to the left of $x_{1}$ and the region to the right of $x_{m}$ both lie in the complement of $\widehat{D}$. Now assume that $\Re\left(P\left(x_{1}\right)\right)<\Re\left(x_{m}\right)$. Since $P(\widehat{D})$ must be contained in the complement of $\widehat{D}$, we must have that, for some $k<m, P\left(x_{1}\right)=x_{k}$. Since the region to the right of $x_{1}$ lies in $\widehat{D}$, and the region to the left of $x_{m}$ lies in $\widehat{D}$, we cannot have that $P\left(x_{1}\right)=x_{m-1}$. Hence $k<m-1$, from which it follows that $P\left(x_{2}\right)=x_{k+1}$. Since each $\widehat{C}_{j}$ and each $\widehat{C}_{j}^{\prime}$ has at most one point of intersection with the line $\left\{\Im(z)=\alpha_{0}\right\}$, we now have that two of the identifications of the $\widehat{C}_{j}$ 
with the $\widehat{C}_{j}^{\prime}$ are accomplished with either $P$ or $P^{-1}$, contradicting the fact that these identifications are free generators of the renormalized $\widehat{G}$.

The above argument also shows that either $P\left(y_{1}\right)=y_{m}$, or $\Re\left(P\left(y_{1}\right)\right)>\Re\left(y_{m}\right)$. Of course, we must have that $P\left(y_{1}\right)=y_{m}$ if and only if $P\left(x_{1}\right)=x_{m}$.

In the case that $P\left(x_{1}\right)=x_{m}$, we define, for $k=1, \ldots, m$, the points

$$
x_{k}^{\prime}=\frac{k-1}{m-1}+i \alpha \quad \text { and } \quad y_{k}^{\prime}=\frac{k-1}{m-1}-i \alpha .
$$

In the case that $\Re\left(P\left(x_{1}\right)\right)>\Re\left(x_{m}\right)$, we define, for $k=1, \ldots, m$, the points

$$
x_{k}^{\prime}=\frac{k-1}{m}+i \alpha \quad \text { and } \quad y_{k}^{\prime}=\frac{k-1}{m}-i \alpha .
$$

We next observe that if $\widehat{C}_{j}$ intersects the line $\left\{\Im(z)=\alpha_{0}\right\}$ at $x_{k}$, then it also intersects the line $\left\{\Im(z)=-\alpha_{0}\right\}$ at $y_{k}$, for no pair of the loops, $\widehat{C}_{j}, \widehat{C}_{i}, \widehat{C}_{i}^{\prime}, \widehat{C}_{j}^{\prime}$, or any of their translates, cross at $\infty$.

We are now in a position to choose the deformed $\widehat{C}_{j}$ and $\widehat{C}_{j}^{\prime}$ as follows. If $\widehat{C}_{j}$ intersects the line $\left\{\Im(z)=\alpha_{0}\right\}$ at say $x_{k}$, then we deform it so that it is linear from $x_{k}$ to $x_{k}^{\prime}$, then linear, with the same real part, to $\infty$, then linear with the same real part to $y_{k}^{\prime}$, then linear to $y_{k}$.

The vertical projection of these deformed loops consists of replacing each of the above pairs of half-infinite lines (i.e., the lines going from some $x_{k}^{\prime}$ to $\infty$, or from some $y_{k}^{\prime}$ to $\left.\infty\right)$ with a trio of line segments, all with the same real part. Two of these line segments keep the line $\{\Re(z)=x\}$ fixed, and run up the vertical sides of the shoebox, one at the point $x+i \alpha$, the other at the point $x-i \alpha$. Up to direction, the lines are then defined as $\{z=x+i \alpha, 0 \leq t \leq \alpha\}$ and $\{z=x-i \alpha, 0 \leq t \leq \alpha\}$. The third line segment runs along the horizontal part of the boundary and can be described as $\{\Re(z)=x,-\alpha \leq \Im(z) \leq \alpha, t=\alpha\}$.

Observe that the vertical projection of the defining loops $\widehat{C}_{1}, \ldots, \widehat{C}_{p}^{\prime}$ for $\widehat{G}$ yields a set of defining loops $C_{1}^{\alpha}, \ldots,\left(C_{p}^{\prime}\right)^{\alpha}$, with corresponding generators $a_{1}=$ $f^{\alpha} \widehat{a}_{1}\left(f^{\alpha}\right)^{-1}, \ldots, a_{p}=f^{\alpha} \widehat{a}_{p}\left(f^{\alpha}\right)^{-1}$ for the Schottky group $G^{\alpha}$. For each fixed choice of the transformations $h_{1}, \ldots, h_{q}$ and each fixed choice of $\alpha_{0}$, where $\alpha_{0}$ is chosen as above, we define the relative conical neighborhood of $\widehat{G}$ to be the set of all marked Schottky groups $G^{\alpha}=\left\langle a_{1}, \ldots, a_{p}\right\rangle$.

We will assume the above normalizations from now on. That is, we normalize $\widehat{G}$ so that $\infty$ is an interior point of the flat part corresponding to $\alpha_{0}$, and we normalize each $f^{\alpha}$ so that $f^{\alpha}(z)=z+O\left(|z|^{-1}\right)$ near $\infty$. With these normalizations, $f^{\alpha} \rightarrow I$ uniformly on compact subsets of $\Omega(\widehat{G})$, and $G^{\alpha} \rightarrow \widehat{G}$ algebraically. We remark that it follows from the Jørgensen-Marden criterion [5] that $G^{\alpha} \rightarrow G$ geometrically.

We also remark that it is unclear how large the relative conical neighborhoods are. However, since each $G^{\alpha}$ is a Schottky group, and, for $\alpha \rightarrow \infty, G^{\alpha} \rightarrow G$, it follows that each relative conical neighborhood contains infinitely many distinct marked Schottky groups. It is also easy to see, as in 11], that, for each primitive parabolic element $\widehat{g} \in \widehat{G}$, as $\alpha \rightarrow \infty$, there is a corresponding geodesic on $S^{\alpha}=\Omega\left(G^{\alpha}\right) / G^{\alpha}$ whose length tends to zero. It follows that each relative conical neighborhood of a noded Schottky group contains Schottky groups representing infinitely many distinct Riemann surfaces. 


\section{Sufficiently COMPlichted NODED SchottKy GRoups}

3.1. Pinchable sets of geodesics. Let $\widehat{G}$ be a noded Schottky group (of genus $p \geq 2$ ) defined by the set of defining loops $\widehat{C}_{1}, \ldots, \widehat{C}_{p}^{\prime}$, with corresponding generators $\widehat{a}_{1}, \ldots, \widehat{a}_{p}$. This set of generators defines a representation of the free group on $p$ elements, $\mathcal{F}_{p}$, into $\operatorname{PSL}(2, \mathbb{C})$; the set of elements of the free group mapped onto parabolic elements of $\widehat{G}$ are the pinched elements. Let $G$ be a Schottky group with defining loops $C_{1}, \ldots, C_{p}^{\prime}$ and generators $a_{1}, \ldots, a_{p}$, where these generators for $G$ lie in one of the relative conical neighborhoods of the generators $\widehat{a}_{1}, \ldots, \widehat{a}_{p}$ for $\widehat{G}$ given in Section 2.2 and where these defining loops have been obtained from $\widehat{C}_{1}, \ldots, \widehat{C}_{p}^{\prime}$ using vertical projection (see 2.2). Let $S=\Omega(G) / G$ be the closed Riemann surface of genus $p$ represented by $G$, and let $V_{i}$ be the projection of $C_{i}, i=1, \ldots, p$. Then $V_{1}, \ldots, V_{p}$ is a set of $p$ homologically independent simple disjoint loops on $S$. Let $\psi: G \rightarrow \widehat{G}$ be the isomorphism defined by $a_{i} \mapsto \widehat{a}_{i}, i=1, \ldots, p$. It is essentially immediate that there are simple disjoint geodesics $L_{1}, \ldots, L_{q}$ on $S$, defined by the words $W_{1}, \ldots, W_{q}$ in the generators $a_{1}, \ldots, a_{p}$, so that $\psi\left(W_{1}\right), \ldots, \psi\left(W_{q}\right)$ are all parabolic in $\widehat{G}$, and every parabolic element of $\widehat{G}$ is a power of a conjugate of one of these.

We remark that the construction in [1] shows that, given the noded Schottky group $\widehat{G}$ with its set of generators, we can choose the parameter $\alpha$, which determines the Schottky group $G$ with its corresponding set of generators, and we can choose the $L_{i}$ on $\Omega(G) / G$ so that the $L_{i}$ are all arbitrarily short. It was further shown in [12] (see also Yamamoto [17]) that if $G$ is any Schottky group with generators $a_{1}, \ldots, a_{p}$, and $L_{1}, \ldots, L_{q}$ is any set of simple disjoint geodesics on $S$ defined by the words $W_{1}, \ldots, W_{q}$, as above, where no $W_{i}$, as an element of $G$, is a non-trivial power, and, for $i \neq j$, the cyclic subgroups generated by $W_{i}$ and $W_{j}$ are not conjugate in $G$, then there is a noded Schottky group $\widehat{G}$, and there is an isomorphism $\psi: G \rightarrow \widehat{G}$, where $\psi\left(W_{1}\right), \ldots, \psi\left(W_{q}\right)$, and their powers and conjugates are exactly the parabolic elements of $\widehat{G}$. More precisely, it was shown in [12] that there is a path in Schottky space, $\mathcal{S}_{\text {alg }}$, which converges to a set of generators for $\widehat{G}$, along which the lengths of the geodesics $L_{1}, \ldots, L_{q}$ all tend to zero.

We now fix a Schottky covering $\Omega(G) \rightarrow S$ of a closed Riemann surface $S$, and we fix a set of generators $a_{1}, \ldots, a_{p}$ for $G$. If $L_{1}, \ldots, L_{q}$ is any set of simple disjoint geodesics on $S$ where no $L_{i}$, as an element of $G$, is a non-trivial power, and $L_{i}$ and $L_{j}$ generate non-conjugate cyclic subgroups of $G$, for all $i \neq j$, then we say that this set of geodesics is pinchable.

Proposition 3.1. Given a non-empty set of $k<3 p-3$ pinchable geodesics, $L_{1}, \ldots, L_{k}$ on $S$, there is a set of $k+1$ pinchable geodesics, $L_{1}, \ldots, L_{k}, L_{k+1}$.

Proof. Since $L_{1}, \ldots, L_{k}$ is pinchable, we can find a noded Schottky group $\widehat{G}$ for which these are pinched. Since $k<3 p-3$, one of the components of $\widehat{G}$, call it $\Delta$, is such that $\widehat{S}=\Delta / \operatorname{Stab}(\Delta)$ is not a thrice-punctured sphere. Let $H=\operatorname{Stab}(\Delta) \subset \widehat{G}$. By Ahlfors' finiteness theorem, since $\Delta$ is a component subgroup of the finitely generated group $\widehat{G}, H$ is finitely generated. Since $H$ is a subgroup of the free group $\widehat{G}$, it is also free. We first take up the case that $\Delta$ is simply connected, in which case $\widehat{H}$ is a quasifuchsian group of the first kind. In this case, since $H$ is free, we can find generators for $H, A_{1}, B_{1}, \ldots, A_{q}, B_{q}, P_{1}, \ldots, P_{r}$, where these generators all 
represent simple loops on $\widehat{S}$, and where the $A_{i}$ and $B_{i}$ are loxodromic (including hyperbolic), the $P_{i}$ are parabolic, these generators satisfy the one defining relation $\left[A_{1}, B_{1}\right] \cdots\left[A_{q}, B_{q}\right] \cdot P_{1} \cdots P_{r}=1$, and every parabolic element of $H$ is conjugate to a power of some $P_{i}$. If $q>0$, then $A_{1}$ is pinchable; if $q=0$, then $r \geq 4$ and $P_{1} P_{2}$ is loxodromic and pinchable. If $\Delta$ is not simply connected, then we can write $H$ as a free product, in the sense of combination theorems, $H=H_{1} * \cdots * H_{q} * F_{r}$, where each of $H_{1}, \ldots, H_{q}$ is a quasifuchsian group, and $F_{r}$ is a Schottky or Schottky-type group. We first take up the case that $q=0$; this is the case that $\Delta=\Omega(H)$. In this case, $G$ is the free product, in the sense of combination theorems, of $p$ infinite cyclic groups. If one of these cyclic groups is loxodromic, then we can pinch the corresponding generator. If all $p$ generators are parabolic, call them $P_{1}, \ldots, P_{p}$, then we can pinch either $P_{1} P_{2}$ or $P_{1} P_{2}^{-1}$. We next take up the case that $q>0$. If some $H_{i}$ does not represent a thrice-punctured sphere, then we proceed as above. If every $H_{i}$ represents a thrice-punctured sphere and $q>1$, then we find geometric generators (i.e., parabolic generators as above) $P_{1,1}, P_{1,2}$ and $P_{1,3}$ for $H_{1}$, and parabolic generators $P_{2,1}, P_{2,2}$ and $P_{2,3}$ for $H_{2}$ as above. That is, $P_{i, 1} P_{i, 2} P_{i, 3}=1$, $i=1,2$. Then the free group $H$ has rank at least 4 , and either $P_{1,1} P_{2,1}$ or $P_{1,1} P_{2,1}^{-1}$ is a primitive loxodromic element of $H$ which represents a simple loop; hence it is pinchable. If $q=1$ and $H_{1}$ represents a thrice punctured sphere, then since $H_{1} \neq H, r \geq 1$. If $F_{r}$ has at least one loxodromic generator, then that generator is pinchable. Otherwise, we write the generators of $H_{1}$ as $P_{1,1}, P_{1,2}$ and $P_{1,3}$ as above, and let $P$ be one of the parabolic generators of $F_{r}$. Then either $P_{1,1} P$ or $P_{1,1} P^{-1}$ represents a simple loop on $\widehat{S}$, and so is pinchable.

3.2. Valid sets of defining loops. Let the Schottky group $G$ uniformizing the Riemann surface $S$ be as above. Let $L_{1}, \ldots, L_{q}$ be a pinchable set of geodesics on $S$, and let $\widehat{S}^{+}$be the noded Riemann surface obtained from $S$ by pinching these $q$ geodesics, $\widehat{S}^{+}$consists of a finite number of compact Riemann surfaces, called parts, which are joined together at a finite number of nodes. Also, let $\widehat{G}$ be the noded Schottky group obtained from $G$ by pinching these $q$ geodesics, let $\widehat{\Omega}$ be the set of discontinuity of $\widehat{G}$, and let $\widehat{\Omega}^{+}$be the noded set of discontinuity of $\widehat{G}$. Let $V_{1}, \ldots, V_{p}$ be a set of defining loops on $S$ and let $\widehat{V}_{1}, \ldots, \widehat{V}_{p}$ be the loops on $\widehat{S}^{+}$ obtained by pinching $L_{1}, \ldots, L_{q}$. We observe that the lifts of the $\widehat{V}_{i}$ to $\widehat{\Omega}^{+}$are all loops, but they are generally not disjoint, and they need not be simple. There are certainly some of these lifts passing through each parabolic fixed point, and some of them might pass more than once through the same parabolic fixed point. The set of loops $V_{1}, \ldots, V_{p}$ is a valid set of defining loops for $L_{1}, \ldots, L_{q}$ if every lift of every $\widehat{V}_{i}$ to $\widehat{\Omega}^{+}$is a simple loop; that is, it passes at most once through each parabolic fixed point. In this case, we note that the set of loops $\widehat{V}_{1}, \ldots, \widehat{V}_{p}$ forms a set of defining loops for $\widehat{G}$ on $\widehat{S}^{+}$.

We note that there are exactly $q$ equivalence classes of parabolic fixed points in $\widehat{G}$, one for each of the loops $L_{i}$. Assume that $V_{1}, \ldots, V_{p}$ is not a valid set of defining loops for $L_{1}, \ldots L_{q}$. Then there is a lift of some $\widehat{V}_{i}$ that passes through a parabolic fixed point more than once. It follows that the corresponding loop $V_{i}$ must cross the corresponding geodesic $L_{j}$ more than once. Further, one sees by opening up the parabolic fixed point into a hyperbolic transformation and looking at the lift of the corresponding $L_{j}$ as being a short path between the fixed points that, in this case, there are necessarily two points of intersection of $V_{i}$ with $L_{j}$, and that there 
is an arc of $L_{j}$ between these two points, so that not only does $V_{i}$ lift to a loop in $\Omega$, but both loops formed by cutting and pasting $V_{i}$ with this arc of $L_{j}$ lift to loops in $\Omega$.

Proposition 3.2. There is at least one valid set of defining loops $V_{1}, \ldots, V_{p}$ for every set of pinchable geodesics $L_{1}, \ldots, L_{q}$.

Proof. Suppose $V_{1}, \ldots, V_{p}$ is a set of defining loops on $S$ so that the corresponding set of defining loops $\widehat{V}_{1}, \ldots, \widehat{V}_{p}$ on $\widehat{S}$ is not valid. Then there is a lift, $\tilde{L}_{i}$, of some $L_{i}$, and there is a lift $\tilde{V}_{j}$ of some $V_{j}$, so that $\tilde{L}_{i}$ and $\tilde{V}_{j}$ cross more than once. We construct a new loop; call it $\tilde{W}$ in $\Omega(G)$ as follows. Start at a point of intersection $x$ of $\tilde{L}_{i}$ and $\tilde{V}_{j}$, and follow the loop $\tilde{V}_{j}$ in one of the two possible directions from $x$ until it again intersects $\tilde{L}_{i}$ at the point $y$; then follow $\tilde{L}_{i}$ back to $x$. Denote by $A$ the arc of $\tilde{L}_{i}$ between $x$ and $y$. Observe that if $A$ intersects some lifting of some $V_{m}$, then, since that lifting of $V_{m}$ is a loop that is disjoint from $\tilde{V}_{j}$, this lifting of $V_{m}$ must cross $A$ an even number of times. Since $A$ is a compact subset of $\Omega(G)$, it can have only a finite number of points of intersection with liftings of the defining loops. Hence, there is a pair of crossing points of $A$ with some lift $\tilde{V}_{k}$ of some $V_{k}$, so that there are no other crossing points of $A$ with any lift of any defining curve between these two. We now replace $\tilde{V}_{k}$ by cutting it at these two crossing points and replacing it by following parallel paths on either side of $A$. After this cut and paste operation, we have replaced $\tilde{V}_{k}$ by either one or two loops, depending on whether the two crossings of $\tilde{V}_{k}$ with $A$ occur with the same or opposite orientations. We then replace $V_{k}$ by these one or two loops, and observe that we now have either $p$ or $p+1$ simple disjoint loops on $S$ that lift to loops; it is easy to observe that $p$ of these loops must be homologically independent.

If we have replaced $V_{k}$ by one loop, there is nothing further to be said. If we have replaced it by two loops, then we observe that we now have $p+1$ loops on $S$ defining the Schottky covering. That is, we now have $p+1$ simple disjoint loops which lift to loops, where every loop that lifts to a loop is freely homotopic to some product of these. It is well known that one of these $p+1$ loops is necessarily redundant. In any case, we have shown that we can find a new set of defining loops for this Schottky covering, where the total number of crossing points of the defining loops with the set of pinchable loops $L_{1}, \ldots, L_{q}$ has been decreased by two.

We remark that the above operation replaces one set of defining loops by another, and so might change the generators of the Schottky group.

3.3. Sufficiently complicated pinchable sets of geodesics. For any valid set of defining loops $V_{1}, \ldots, V_{p}$, we define the complexity as follows. First, we can deform the $V_{i}$ on $S$ so that they are all geodesics. Then the geometric intersection number $V_{i} \bullet L_{j}$ of $V_{i}$ with $L_{j}$ is well defined; it is the number of points of intersection of these two geodesics. Looking on the corresponding noded surface $\widehat{S}^{+}, V_{i} \bullet L_{j}$ is the number of times the curve $\widehat{V}_{i}$, obtained from $V_{i}$ by contracting $L_{j}$ to a point, passes through that point (node). The complexity of $V_{1}, \ldots, V_{p}$ is then

$$
\max \sum_{i=1}^{p} V_{i} \bullet L_{j},
$$

where the maximum is taken over all $j=1, \ldots, q$. The complexity $\Xi\left(L_{1}, \ldots, L_{q}\right)$ is the minimum of the complexities of $V_{1}, \ldots, V_{p}$, where the minimum is taken 
over all valid sets of defining loops. The crucial point of the complexity is that if $\Xi\left(L_{1}, \ldots, L_{q}\right) \geq n$, then, for every valid defining set $V_{1}, \ldots, V_{p}$, there is a node $P$ on $S^{+}$so that the total number of crossings of $P$ by $\widehat{V}_{1}, \ldots, \widehat{V}_{p}$ is at least $n$. Now assume that $q=3 p-3$, so that $\widehat{G}$ is a maximal noded Schottky group. Observe that $\widehat{G}$ is rigid, and that every part of $S^{+}$is a sphere with three distinct nodes. Also, every connected component $\Delta \subset \widehat{\Omega}$ is a Euclidean disc $\Delta$, where $\Delta / \operatorname{Stab}(\Delta)$ is a sphere with three punctures - the three punctures correspond to the three nodes of the corresponding part of $\widehat{S}^{+}$. Let $V_{1}, \ldots, V_{p}$ be a valid set of defining loops on $S$, and let $\widehat{V}_{1}, \ldots, \widehat{V}_{p}$ be the corresponding loops on $\widehat{S}^{+}$. For each $i=1, \ldots, p$, the intersection of a lifting of $\widehat{V}_{i}$ with a component of $\widehat{G}$ (i.e., a connected component of $\widehat{\Omega}$ ) is called a strand of that lifting $\widehat{V}_{i}$. Similarly, the loops $\widehat{V}_{1}, \ldots, \widehat{V}_{p}$ appear on the corresponding parts of $\widehat{S}^{+}$as collections of strands connecting the nodes on the boundary of each part. There are two possibilities for these strands - either a strand connects two distinct nodes on some part, or it starts and ends at the same node.

Since the loops $V_{1}, \ldots, V_{p}$ are simple and disjoint, there are at most three sets of parallel strands of the $\widehat{V}_{i}$ in each part; that is, there are at most three sets of strands, where any two strands in the same set are homotopic arcs with fixed endpoints at the nodes. We regard each of these sets of strands on a single part as being a superstrand, so that there are at most 3 superstrands on any one part.

We next look in some component $\Delta$ of $\widehat{G}$, and look at a parabolic fixed point $x$ on its boundary, where $x$ corresponds to the node $N$ on the part $S_{i}$ of $\widehat{S}^{+}$. In general, there will be infinitely many liftings of superstrands emanating from $x$ in $\Delta$, but, modulo $\operatorname{Stab}(\Delta)$, there are only finitely many. In fact, there are at most 4 such liftings of superstrands emanating from $x$. If there is exactly one superstrand on $S_{i}$ with one endpoint at $N$, and the other endpoint at a different node, then modulo $\operatorname{Stab}(\Delta)$ there will be exactly the one lifting of this superstrand emanating from $x$. If there is only one superstrand on $S_{i}$ with both endpoints at the same node $N$, then this superstrand has two liftings starting at $x$, one in each direction; so, in this case, we see two lifts of superstrands modulo $\operatorname{Stab}(\Delta)$ emanating from $x$. It follows that, modulo $\operatorname{Stab}(\Delta)$, we can have $0,1,2,3$ or 4 liftings of superstrands starting at $x$. We note that these liftings of superstrands all end at distinct parabolic fixed points on the boundary of $\Delta$.

The defining set of loops $\widehat{V}_{1}, \ldots, \widehat{V}_{p}$ is sufficiently complicated if there are two (different) lifts $\widehat{C}_{i}$ and $\widehat{C}_{j}$ of some $\widehat{V}_{i}$ and some not necessarily distinct $\widehat{V}_{j}$, respectively, so that $\widehat{C}_{i}$ and $\widehat{C}_{j}$ both pass through the parabolic fixed point $z_{1}$ into a component $\Delta_{1}$ of $\widehat{G}$. Then $\widehat{C}_{i}$ and $\widehat{C}_{j}$ both travel through $\Delta_{1}$ to the same parabolic fixed point on its boundary, $z_{2}$, and into another component $\Delta_{2}$, in which they again traverse together to the same boundary point, $z_{3}$, necessarily a parabolic fixed point, where they enter $\Delta_{3}$ and leave $\Delta_{3}$ at different parabolic fixed points.

3.4. Sufficiently complicated noded Schottky groups. A noded Schottky group $\widehat{G}$ is sufficiently complicated if every set of valid defining loops on $\widehat{S}^{+}$is sufficiently complicated. We note that (keeping the notation of last subsection) inside $\Delta_{1}, \widehat{C}_{i}$ and $\widehat{C}_{j}$ are disjoint: they both enter $\Delta_{1}$ at the same point, and they both leave $\Delta_{1}$ at the same point; hence they cannot both be circles. This idea will 
be explored further in Section 6 However, we need the full strength of the hypothesis of sufficiently complicated Schottky groups to prove that no nearby Schottky group can be classical.

Proposition 3.3. If a maximal noded Schottky group $\widehat{G}$ has complexity at least 11 , then it is sufficiently complicated.

Proof. Let $V_{1}, \ldots, V_{p}$ be a valid set of defining loops for $\widehat{G}$. Then, since the complexity $n \geq 11$, there is a node $N \subset \widehat{S}^{+}$that is crossed at least $n$ times by $\widehat{V}_{1}, \ldots, \widehat{V}_{p}$. Let $S_{1}$ and $S_{2}$ be the not necessarily distinct spheres with three nodes on either side of the node $N$. Consider the finite set of $n$ strands in $S_{1}$, starting at the node $N$, defined by $\widehat{V}_{1}, \ldots, \widehat{V}_{p}$-call these strands $A_{1}, \ldots, A_{n}$. Ignoring direction, they separate into at most three superstrands (i.e., sets of parallel strands), each with a distinct endpoint; however, one of these superstrands might start and end at the same endpoint, $N$. Normalize the universal covering of $S_{1}$ so that it is the upper half-plane $\mathbb{H}^{2}$, and so that $N$ is the projection of the point at infinity; assume that the stabilizer of $\infty$ is generated by $P(z)=z+1$. Choose some lifting $\widetilde{A}_{1,0}$ of $A_{1}$, starting at $\infty$, and consider the set $\widetilde{A}$ of all liftings of all the $A_{i}$ starting at $\infty$, and lying between $\widetilde{A}_{1,0}$ and its image under $z \mapsto z+1$. As observed above, we can choose $\widetilde{A}_{1,0}$ so that $\widetilde{A}$ will divide into at most four superstrands, where each of these four superstrands has a distinct endpoint on the circle at infinity of $\mathbb{H}^{2}$. We call these four superstrands $\widetilde{A}_{1}, \ldots, \widetilde{A}_{4}$; we will give an explicit ordering below. We write the universal covering of $S_{2}$ as $\widetilde{\mathbb{H}^{2}}$, which we can think of as being the set $\Im(z)<\alpha<0$, with $\infty$ projecting to $N$. (Note that even if $S_{1}=S_{2}$, following the lifting of a path through the node $N$, we travel from one representation of the universal covering of $S_{1}$ to another.) Each of the strands in $S_{1}$ has a unique continuation in $S_{2}$, so that when we look in $\mathbb{H}^{2} \cup \widetilde{\mathbb{H}^{2}}$, we see a set of $n$ arcs, each starting at some point on the real axis, passing through infinity, and ending at some point on the boundary of $\widetilde{\mathbb{H}^{2}}$. The crucial point is that these arcs touch without crossing at $\infty$. For each $i=1, \ldots, 4$, the continuations of the strands of $\widetilde{A}_{i}$ separate into some number, call it $m_{i}$, of superstrands in $\widetilde{\mathbb{H}^{2}}$. As above, $m_{i} \leq 4$. We now order the $\widetilde{A}_{i}$ so that $m_{1} \geq \cdots \geq m_{4}$. It is easy to observe that if $m_{1}=4$, then, for $i=2,3,4, m_{i} \leq 2$. Similarly, if $m_{1}=3$, then $m_{2} \leq 3, m_{3} \leq 2$ and $m_{4} \leq 2$. In any case, we have that $m_{3} \leq 2$ and $m_{4} \leq 2$. Now let $\left|\widetilde{A}_{i}\right|$ be the number of strands in $\widetilde{A}_{i}$; we are given that $\left|\widetilde{A}_{1}\right|+\left|\widetilde{A}_{2}\right|+\left|\widetilde{A}_{3}\right|+\left|\widetilde{A}_{4}\right|=n \geq 11$. This set of defining loops is sufficiently complicated if there are two strands of some $\widetilde{A}_{i}$ whose continuations in $\widetilde{\mathbb{H}^{2}}$ are also parallel. We now assume that this set of defining loops is not sufficiently complicated. There are several cases to consider. We first assume that $m_{1}=4$; then $m_{j} \leq 2, j=2,3,4$, from which it follows that $\left|\widetilde{A}_{1}\right|=4,\left|\widetilde{A}_{2}\right| \leq 2,\left|\widetilde{A}_{3}\right| \leq 2$ and $\left|\widetilde{A}_{4}\right| \leq 2$, so that $\left|\widetilde{A}_{1}\right|+\left|\widetilde{A}_{2}\right|+\left|\widetilde{A}_{3}+\right| \widetilde{A}_{4} \mid \leq 10$. If $m_{1}=3$, then $m_{2} \leq 3$, $m_{3} \leq 2$ and $m_{4} \leq 2$. Then $\left|\widetilde{A}_{1}\right|=3,\left|\widetilde{A}_{2}\right| \leq 3,\left|\widetilde{A}_{3}\right| \leq 2$ and $\left|\widetilde{A}_{4}\right| \leq 2$, so that $\left|\widetilde{A}_{1}\right|+\left|\widetilde{A}_{2}\right|+\left|\widetilde{A}_{3}\right|+\left|\widetilde{A}_{4}\right| \leq 10$. If $m_{1}=2$, then $m_{2} \leq 2, m_{3} \leq 2$ and $m_{4} \leq 2$, from which it follows, as above, that $\left|\widetilde{A}_{1}\right|+\left|\widetilde{A}_{2}\right|+\left|\widetilde{A}_{3}\right|+\left|\widetilde{A}_{4}\right| \leq 8$. Finally, if $m_{1}=1$, then $\left|\widetilde{A}_{1}\right|+\left|\widetilde{A}_{2}\right|+\left|\widetilde{A}_{3}\right|+\left|\widetilde{A}_{4}\right| \leq 4$.

Proposition 3.4. For each positive integer $n$, and for each fixed genus $p \geq 2$, there are only finitely many topologically distinct maximal noded Schottky groups of genus $p$ and complexity $n$. 
Proof. Fix a Schottky group with defining loops $C_{1}, \ldots, C_{p}^{\prime}$, with a set of pinchable curves on the underlying surface, $S$. Let $V_{1}, \ldots, V_{p}^{\prime}$ be the projections of the defining loops to $S$, and let $L_{1}, \ldots, L_{q}$ be a set of pinchable loops, where the number of points of intersection of each $V_{i}$ with each $L_{j}$ is the geometric intersection number. We assume that these loops realize the complexity; that is, $\sum_{j=1}^{p} V_{1} \bullet L_{j}=n$, and, for every $i>1, \sum_{j=1}^{p} V_{i} \bullet L_{j} \leq n$. Consider all lifts of the $V_{i}$ to the fundamental domain defined by the defining curves, and contract the defining curves $C_{1}, \ldots, C_{p}^{\prime}$ to points. This yields a planar graph with $2 p$ vertices and $m$ edges, where

$$
m=\sum_{i} \sum_{j} V_{i} \bullet L_{j} .
$$

Since the genus of the underlying surface is $p$, there are at most $3 p-3$ pinchable curves, from which it follows that $m \leq(3 p-3) n$. It is clear that if two such planar graphs with the same pairing of the vertices, including the identifications of the edges at each pair of vertices, are topologically equivalent, then the corresponding noded Schottky groups are topologically equivalent. Our result now follows from the obvious fact that there are only finitely many topologically distinct planar graphs with $2 p$ vertices and $m$ edges.

Proposition 3.5. For every genus $p \geq 2$, there are infinitely many topologically distinct geometrically finite maximal noded Schottky groups of genus $p$.

Proof. Let $S_{0}$ be a surface of genus 2 , and let $A_{1}, B_{1}, A_{2}, B_{2}$ be a standard homotopy basis on $S_{0}$. Let $N$ be the smallest normal subgroup of $\pi_{1}\left(S_{0}\right)$ containing $A_{1}$ and $A_{2}$. Then we can regard the corresponding Schottky group $G_{0}$ as being generated by the elements $b_{1}$ and $b_{2}$ corresponding to $B_{1}$ and $B_{2}$, respectively. We first replace $B_{1}$ and $B_{2}$ by their corresponding geodesics. We then observe that the geodesic corresponding to the element $\left(B_{1} B_{2}\right)^{n} B_{1} A_{1} B_{1}^{-1} A_{1}^{-1}\left(B_{2} B_{1}\right)^{-n}$ is simple and disjoint from the geodesics corresponding to $B_{1}$ and $B_{2}$. The corresponding word $W_{n}$ in the free group generated by $b_{1}$ and $b_{2}$ is then $w_{n}=\left(b_{1} b_{2}\right)^{n}\left(b_{2} b_{1}\right)^{-n}$. This word is obviously cyclically reduced and primitive. Hence the triple, $\left(b_{1}, b_{2}, w_{n}\right)$, is pinchable. Since the length $\left|w_{n}\right|=4 n$, these triples are all distinct. Since $b_{1}$ and $b_{2}$ are generators, and $w_{n}$ cannot be a free generator, no element of $\operatorname{Aut}\left(\mathcal{F}_{2}\right)$ can map the triple $\left(b_{1}, b_{2}, w_{n}\right)$ onto the triple $\left(b_{1}, b_{2}, w_{m}\right)$ unless $m=n$. For genus 2, the desired result follows at once; for higher genus, the result follows from Proposition 3.1 .

Proposition 3.6. For every genus $p \geq 2$, there are infinitely many sufficiently complicated maximal noded Schottky groups of genus $p$.

Proof. This is an immediate consequence of the above three propositions.

Proposition 3.7. There are only finitely many topologically distinct maximal neoclassical noded Schottky groups.

Proof. Let $C_{1}, \ldots, C_{p}^{\prime}$ be defining circles for a maximal neoclassical noded Schottky group. Each of the $2 p$ circles can intersect any of the other circles in at most one point, so there is a bound on the number of parabolic fixed points on the boundary of the fundamental domain defined by the common exterior of these circles. Except for the fact that we cannot tell the difference between an element and its inverse, the parabolic element fixing each of these points can be uniquely written as a word $W$ in the generators, $a_{1}, \ldots, a_{p}$ (see [14]). One can describe this word $W$ as 
follows. Start with a point of tangency $x=x_{1}$ of say $C_{i}$ and $C_{j}$, then $a_{j}$ maps $x_{1}$ to a point of tangency $x_{2}=a_{j}\left(x_{1}\right)$ of $C_{j}^{\prime}$ with some $C_{k}$ or $C_{k}^{\prime}$. If it is $C_{k}$, then $a_{k}\left(x_{2}\right)=a_{k} a_{j}(x)$ is a point of tangency of $C_{k}^{\prime}$ with some other circle; if it is $C_{k}^{\prime}$, then $a_{k}^{-1}\left(x_{2}\right)=a_{k}^{-1} a_{j}(x)$ is a point of tangency of $C_{k}$ with some other circle. Continuing in this manner, we eventually return to $x$, and so generate the word $W=\ldots a_{k} a_{j}$ or $W=\ldots a_{k}^{-1} a_{j}$, which fixes $x$. We remarked above, and it was shown in 14, that every parabolic element of $G$ is a power of a conjugate of one of the parabolic elements whose fixed point lies on the intersection of two of the defining loops. Since any two of the $2 p$ circles $C_{1}, \ldots, C_{p}^{\prime}$ has at most one point of tangency, the word $W$ can contain a juxtaposed pair of generators $a_{i} a_{j}$, or $a_{i}^{-1} a_{j}$, or $a_{i} a_{j}^{-1}$, or $a_{i}^{-1} a_{j}^{-1}$, at most once. Hence, for any neoclassical noded Schottky group $G$, there is a bound on the lengths of these words defining the pinched elements of $G$. It follows that there is a finite list $G_{1}, \ldots, G_{k}$ of maximal neoclassical groups so that if $G$ is any maximal neoclassical group, then there is a $G_{i}$ in our list, and there is an isomorphism $\phi: G \rightarrow G_{i}$ with the property that $g \in G$ is parabolic if and only if $\phi(g) \in G_{i}$ is parabolic. However, it was shown in [7 that every such isomorphism is in fact a conjugation by either an element of $P S L(2, \mathbb{C})$ or by an orientation reversing conformal homeomorphism of $\widehat{\mathbb{C}}$.

Remark. The hypotheses of the above proposition are too strong; we do not need to assume that the noded Schottky group is maximal. To see this, observe that, since any pair of distinct circles can meet in at most one point, there are only finitely many topologically distinct configurations of $2 p$ circles, all having a common outside. Also, for any two such sets of $2 p$ circles having the same configuration of points of tangency, it is easy to construct a path of topological deformations from one to the other. Hence it suffices to choose one such set of $2 p$ circles for each such configuration. Since we require that every point of tangency be a parabolic fixed point, it follows that each generator $\widehat{a}_{i}$ maps the points of tangency on $\widehat{C}_{i}$ to the points of tangency on $\widehat{C}_{i}^{\prime}$. Finally, we observe that, for each $i$ and each possible choice of pairings of the tangency points on $\widehat{C}_{i}$ with the corresponding tangency points on $\widehat{C}_{i}^{\prime}$, one of the following situations occurs. Either there are at least 3 points of tangency on $\widehat{C}_{i}$, in which case the transformation $\widehat{a}_{i}$ is determined by the pairings of these points of tangency, or there are exactly two points of tangency on $\widehat{C}_{i}$, in which case $\widehat{a}_{i}$ is determined up to a 1-parameter continuous family of hyperbolic motions keeping these two points fixed and keeping $\widehat{C}_{i}$ invariant, or there is exactly one point of tangency on $\widehat{C}_{i}$, in which case $\widehat{a}_{i}$ is determined up to a 1-parameter continuous family of parabolic motions keeping this one point fixed and keeping $\widehat{C}_{i}$ invariant, or there are no points of tangency, in which case $\widehat{a}_{i}$ is determined up to an arbitrary element of the 3-parameter subgroup of $P S L(2, \mathbb{C})$ keeping both discs bounded by $\widehat{C}_{i}$ invariant.

\section{Cross Ratios and inequalities for sufficiently Complicated groups}

If $z_{1}, z_{2}, z_{3}, z_{4}$ are four distinct points on the extended complex plane $\widehat{\mathbb{C}}$, then we denote their cross-ratio by

$$
\left(z_{1}, z_{2} ; z_{3}, z_{4}\right)=\frac{\left(z_{1}-z_{3}\right)\left(z_{2}-z_{4}\right)}{\left(z_{1}-z_{4}\right)\left(z_{2}-z_{3}\right)} .
$$


We will use the well-known facts that elements of $\operatorname{PSL}(2, \mathbb{C})$ preserve the crossratio, and that the cross-ratio is real if and only if the four points lie on a circle. We will usually use the cross-ratio in the normal form

$$
(0, \infty ; z,-i)=i z
$$

We will need the following two easy exercises concerning the level 2 congruence subgroup of the modular group.

Proposition 4.1. Let $H$ be a Fuchsian group representing the thrice-punctured sphere. We assume that $H$ acts on the upper half-plane, and that $H$ has been conjugated so that $z \mapsto z+\alpha, \alpha \geq 1$, is a primitive parabolic element in $H$. Let $E_{1}$ and $E_{2}$ be two complete geodesics starting at $\infty$, where $E_{1}$ and $E_{2}$ end at distinct parabolic fixed points $y_{1}$ and $y_{2}$ of $H$, and where $E_{1}$ and $E_{2}$ project onto complete simple disjoint geodesics on $\mathbb{H}^{2} / H$. Then $\left|y_{1}-y_{2}\right| \geq \frac{|\alpha|}{4}$.

Proof. Since $E_{1}$ and $E_{2}$ are disjoint geodesics, either $y_{1}$ or $y_{2}$ is not $H$-equivalent to $\infty$; we can assume it is $y_{1}$. Conjugate $H$ by a translation so that $y_{1}=0$. Observe that $H$ contains the primitive transformations $z \mapsto z+\alpha$ and $z \mapsto \frac{z}{ \pm \beta z+1}$, where $\beta=\frac{-4}{\alpha}$. Then, since the projection of $E_{2}$ cannot cross the projection of $E_{1}$, $\left|y_{2}\right| \geq \frac{\alpha}{4}$.

For the next proposition, we consider $\widehat{G}_{0}$ as being a maximal noded Schottky group, and we consider the truncated flat part of $B^{\alpha, n}$ as defined in Section 2.2. If $\Delta \subset \Omega\left(\widehat{G}_{0}\right)$, then, for each $\alpha$ and each $n$, we call the intersection of the truncated flat part of $B^{\alpha, n}$ with $\Delta$ the $(\alpha, n)$-compact part of $\Delta$. Up to conjugation, we always may assume that $\Delta$ is the upper half-plane.

Proposition 4.2. Let $H$ be a Fuchsian group, acting on $\mathbb{H}^{2}$ and representing the thrice-punctured sphere, where $H$ is generated by $a(z)=z+\alpha$ and $b(z)=\frac{z}{\beta z+1}$, and where $\alpha \geq 1$ and $\alpha \beta=-4$. Let $R$ be the Euclidean ray through the origin defined by $\arg (z)=\theta, 0<|\theta| \leq \pi / 2$. If $|\theta| \leq \pi / 6$, then $R$ does not project to a simple path on $\mathbb{H}^{2} / H$. Further, given $K>0$ and an $(\alpha, n)$-compact part of the upper half-plane, there is a constant $\theta_{0}, 0<\theta_{0}<\pi / 6$, so that, for all $\theta$ with $0<|\theta|<\theta_{0}$, there are four points, $z_{1}, z_{2}, z_{3}, z_{4}$, in the $(\alpha, n)$-compact part of the upper half-plane, so that

$$
\left|\Im\left(z_{1}, z_{2} ; z_{3}, z_{4}\right)\right| \geq K
$$

Proof. For the first statement, conjugate $H$ by a dilation, so that $\alpha=-\beta=2$. Consider the transformation $(a b)^{-1}(z)=\frac{z-2}{2 z-3}$, and observe that this maps $R$ onto a set disjoint from $R$ precisely when $|\tan \theta|>\frac{1}{\sqrt{3}}$. The second statement follows almost at once from the observation that, as $\theta \rightarrow 0$, the region between the line $\arg (z)=\theta$ and its translate under $b a$ increases, and covers larger and larger portions of any compact part of the upper half-plane.

Proposition 4.3. Let $\widehat{G}$ be a sufficiently complicated maximal noded Schottky group. Then, for any set of defining loops $\widehat{C}_{1}, \ldots, \widehat{C}_{p}^{\prime}$, there are four successive parabolic fixed points $z_{1}, z_{2}, z_{3}, z_{4}$ on some $\widehat{C}_{i}$ so that $\left|\Im\left(z_{2}, z_{3} ; z_{4}, z_{1}\right)\right| \geq \frac{1}{8}$.

Proof. Let $\widehat{C}_{1}, \ldots, \widehat{C}_{p}^{\prime}$ be any set of defining loops for $\widehat{G}$. Since $\widehat{G}$ is sufficiently complicated, there is some $\widehat{C}_{j}$, call it $\widehat{C}$, there is some translate $\widehat{C}^{\prime}$ of some $\widehat{C}_{k}$, and there are three distinct components $\Delta_{1}, \Delta_{2}$ and $\Delta_{3}$ of $\widehat{G}$, so that, after appropriate 
normalization, $\widehat{C}$ and $\widehat{C}^{\prime}$ both enter $\Delta_{1}$ at the parabolic fixed point $-i=z_{1}$. These curves both leave $\Delta_{1}$ and enter $\Delta_{2}$ at the parabolic fixed point $0=z_{2}$, they both leave $\Delta_{2}$ at the parabolic fixed point, $\infty=z_{3}$, where they enter $\Delta_{3}$, and they leave $\Delta_{3}$ at distinct parabolic fixed points, $z_{4}$ and $z_{4}^{\prime}$. We remark that, while $\widehat{C}$ and $\widehat{C}^{\prime}$ are required to be distinct, $j$ is not necessarily distinct from $k$.

Since $\Delta_{2}$ and $\Delta_{3}$ are disjoint, and both have $\infty$ on their boundary, their boundaries in $\mathbb{C}$ consist of two parallel lines; the boundary of $\Delta_{2}$ passes through the origin. It then follows that $\Delta_{1}$, which is disjoint from both $\Delta_{2}$ and $\Delta_{3}$, is a Euclidean disc of finite diameter; let $d$ denote the diameter of $\Delta_{1}$.

Let $g(z)=z+\alpha$ be a generator of $\operatorname{Stab}(\infty) \subset \operatorname{Stab}\left(\Delta_{2}\right)$; then $g$ is also a generator of $\operatorname{Stab}(\infty)$ in $\operatorname{Stab}\left(\Delta_{3}\right)$. Since $g\left(\Delta_{1}\right) \neq \Delta_{1}$, we must have that $g\left(\Delta_{1}\right) \cap \Delta_{1}=\emptyset$; hence $|\alpha| \geq d$. We now write $\Delta_{2}$ as $\{z \mid-\pi / 2+\theta<\arg (z)<\pi / 2+\theta\}$, or, equivalently, $\left\{\Re\left(e^{-i \theta} z\right)>0\right\}$. Since $-i$ is not on the boundary of $\Delta_{2}, 0<\theta<\pi$. The boundary of $\Delta_{3}$ is a line parallel to the boundary of $\Delta_{2}$ and passing through the imaginary axis at some point $y<-1$. Since the boundary of $\Delta_{1}$ is a Euclidean circle passing through the point $-i$ and tangent to $\arg (z)=\pi / 2+\theta$ at the origin, its diameter is given by $d=\csc \theta$. Replacing $g(z)=z+\alpha$ by its inverse if necessary, we can write $\alpha=\rho e^{i(\theta-\pi / 2)}$, where $|\alpha|=\rho \geq d=\csc \theta$. We observe that $\Re(\alpha)=$ $|\alpha| \sin \theta \geq 1$. We conclude from Proposition 4.1 that $\left|z_{4}-z_{4}^{\prime}\right| \geq \frac{|\alpha|}{4}$. It follows that

$$
\left|\Re\left(z_{4}\right)-\Re\left(z_{4}^{\prime}\right)\right|=\left|z_{4}-z_{4}^{\prime}\right| \sin \theta \geq \frac{|\alpha| \sin \theta}{4} \geq \frac{1}{4} .
$$

Our conclusion now follows from the fact that $\Im\left(z_{2}, z_{3} ; z, z_{1}\right)=\Im(0, \infty ; z,-i)=$ $\Re(z)$.

\section{Non-Classical SchottKy Groups}

Let $\widehat{C}_{1}, \ldots, \widehat{C}_{p}^{\prime}$, with generators, $\widehat{a}_{1}, \ldots, \widehat{a}_{p}$, be a set of defining loops for the sufficiently complicated maximal noded Schottky group $\widehat{G}$, and let $G_{n}$ be a sequence of Schottky groups converging to $\widehat{G}$ in a relative conical neighborhood (see Section 2.2). That is, there is some number $\alpha_{0}$, so that the relative conical neighborhood consists of all Schottky groups $G^{\alpha}$ obtained via vertical projection from $\widehat{C}_{1}, \ldots, \widehat{C}_{p}^{\prime}$, where the vertical projection is defined with reference to the infinite shoebox of size $\alpha$. We reiterate that the infinite shoebox, and hence the vertical projection, depend on a choice of conjugating transformations, one for each conjugacy class of maximal parabolic subgroups of $\widehat{G}$.

Theorem 5.1. Let $\widehat{G}$ be a sufficiently complicated maximal noded Schottky group. If $\alpha_{0}$ is sufficiently large, then every Schottky group in the relative conical neighborhood of $\widehat{G}$ defined by $\alpha_{0}$ is not classical.

Proof. We fix some $\alpha_{0}$ so that, using our normalizing transformations, we have the proper precise invariance. That is, the complement of the flat part $B_{0}$, defined by $\alpha_{0}$, is a disjoint union of pairs of tangent Euclidean discs, where each pair is precisely invariant under the parabolic cyclic subgroup with fixed point at the point of tangency. As above, we normalize $\widehat{G}$ so that $\infty$ is a point in the truncated flat part of $B_{0}$; then there is some component $\Delta_{0}$ of $\widehat{G}$ so that $\infty \in \Delta_{0}$. Since $\widehat{G}$ is maximal noded, there are $3 p-3$ parabolic elements $\widehat{g}_{1}, \ldots, \widehat{g}_{3 p-3}$ that generate non-conjugate maximal parabolic cyclic subgroups of $\widehat{G}$. For each $\alpha>\alpha_{0}$, set $g_{i}^{\alpha}=f^{\alpha} \widehat{g}_{i}\left(f^{\alpha}\right)^{-1}$. Fix $\epsilon>0$; we first choose $\alpha_{1}>\alpha_{0}$ so that, for $\alpha>\alpha_{1}$, the fixed 
points of each $g_{i}^{\alpha}$ lie within $\epsilon$ of the fixed point of $\widehat{g}_{i}$. We need the following in order to discuss the twist of the projection of a defining curve near a node. For each fixed $i$, renormalize $\widehat{G}$ so that $\widehat{g}_{i}(z)=z+1$. For $m \in \mathbb{Z}$, we call the lines $\{\Re(z)=m\}$ slope lines. The truncated flat part of $B^{\alpha, n}$ has non-trivial intersection with $2 n+1$ slope lines (two of these are on the boundary). The slope lines are then well defined near every parabolic fixed point of $\widehat{G}$.

The content of Lemma 5.1 below is that, for $\alpha$ sufficiently large, if $C$ is a circular defining loop for $G^{\alpha}$, then the vertical projection of $C$ has only a finite amount of twist as it passes through each node. Now suppose that $G^{\alpha}$ is classical, where $\alpha>$ $\alpha_{2}$, chosen as above. Let $C_{1}, \ldots, C_{p}^{\prime}$ be defining circles for $G^{\alpha}$, and let $\widehat{C}_{1}, \ldots, \widehat{C}_{p}^{\prime}$ be their vertical projections, defined by $\alpha_{0}$. Also, let $n$ be as given in Lemma 5.1. If $\widehat{C}_{1}, \ldots, \widehat{C}_{p}^{\prime}$ is not a set of defining loops for $\widehat{G}$, then there is some $\widehat{C}_{i}$ that passes more than once through the same parabolic fixed point. In this case, it is clear that we can find four points on $\widehat{C}_{i}$ and lying in the truncated flat part of $B^{\alpha_{0}, n}$, so that the imaginary part of their cross-ratio is bounded away from zero, from which it follows that $C_{i}$ cannot be a circle. If $\widehat{C}_{1}, \ldots, \widehat{C}_{p}^{\prime}$ is a set of defining loops for $\widehat{G}$, then, since $\widehat{G}$ is sufficiently complicated, there is some defining curve $\widehat{C}$ so that $\widehat{C}$ enters some component $\Delta_{1}$ at the parabolic fixed point $x_{1}$, it leaves $\Delta_{1}$ and enters $\Delta_{2}$ at the parabolic fixed point $x_{2}$, it leaves $\Delta_{2}$ and enters $\Delta_{3}$ at the parabolic fixed point $x_{3}$, and it leaves $\Delta_{3}$ at the parabolic fixed point $x_{4}$, where, by Proposition $4.3,\left|\Im\left(x_{2}, x_{3} ; x_{4}, x_{1}\right)\right| \geq \frac{1}{8}$. If follows from Lemma 5.2 below that there are in fact a finite number of sets of four parabolic fixed points $\left(x_{1,1}, x_{1,2}, x_{1,3}, x_{1,4}\right), \ldots,\left(x_{N, 1}, x_{N, 2}, x_{N, 3}, x_{N, 4}\right)$ of $\widehat{G}$, so that the following hold:

(i) For every $j=1, \ldots, N$,

$$
\left|\Im\left(x_{j, 2}, x_{j, 3}, x_{j, 4}, x_{j, 1}\right)\right| \geq \frac{1}{8}
$$

and

(ii) for every $\alpha>\alpha_{2}$, and for every classical set of defining loops $C_{1}, \ldots, C_{p}^{\prime}$ of any $G^{\alpha}$, there is some $j, 1 \leq j \leq N$, and there is some $C_{i}$ so that $\widehat{C}$, the vertical projection of this $C_{i}$, passes through the four points $\left(x_{j, 2}, x_{j, 3}, x_{j, 4}, x_{j, 1}\right)$.

We next fix $n$ so that if $C$ is a circular defining loop for some $G^{\alpha}$, with $\alpha>\alpha_{0}$, then the vertical projection of $C$ crosses at most $n$ slope lines. Note that the minimal $n$ satisfying this requirement decreases as $\alpha_{0}$ increases; hence we can increase $\alpha_{0}$ while leaving $n$ constant. We next choose $\alpha_{0}$ sufficiently large, so that, for each one of our finite number of sets of four parabolic fixed points, if $z_{i}$ is a point on the boundary of the flat part of $B^{\alpha_{0}, n}$, where $z_{i}$ lies on the horizontal part of the boundary component near $x_{i}$, then $\left|\Im\left(z_{2}, z_{3} ; z_{4}, z_{1}\right)\right| \geq \frac{1}{16}$. Finally, we choose $\alpha_{1}>\alpha_{0}$ so that for all $\alpha>\alpha_{1}, f^{\alpha}$ is sufficiently close to the identity so that, for all $z_{1}, z_{2}, z_{3}, z_{4}$ as above, $\left|\Im\left(f^{\alpha}\left(z_{2}\right), f^{\alpha}\left(z_{3}\right) ; f^{\alpha}\left(z_{4}\right), f^{\alpha}\left(z_{1}\right)\right)\right| \geq \frac{1}{32}$.

Lemma 5.1. There is an $n \in \mathbb{Z}$ and an $\alpha_{2}>\alpha_{1}$, so that for any $\alpha>\alpha_{2}$ and for any defining loop $C$ for $G^{\alpha}$, where $C$ is a Euclidean circle, the following holds. Let $\widehat{g}$ be a parabolic element of $\widehat{G}$, where the vertical projection of $C$ passes through the fixed point of $\widehat{g}$. Then, near the fixed point of $\widehat{g}$ in the truncated flat part of $B^{\alpha_{0}, n}$, the vertical projection of $C$ passes through fewer than $n$ slope lines.

Proof. We have already observed that $f^{\alpha}$ converges to the identity uniformly on compact subsets of the truncated flat part of $B^{\alpha_{0}, n}$. Then, for $\alpha$ sufficiently large, 
since $C$ is a Euclidean circle, the intersection of $\left(f^{\alpha}\right)^{-1}(C)$ with the truncated flat part of $B^{\alpha_{0}, n}$ is close to a straight line in the spherical metric. However, the content of Proposition 4.2 is that a Euclidean straight line passing through the upper halfplane that projects to a simple arc on $\mathbb{H}^{2} / \Gamma_{0}$ passes through at most finitely many slope lines in the truncated flat part. The desired result now follows.

Lemma 5.2. There is a finite set of quadruples of parabolic fixed points $\left(x_{1,1}, x_{1,2}\right.$, $\left.x_{1,3}, x_{1,4}\right), \ldots,\left(x_{N, 1}, x_{N, 2}, x_{N, 3}, x_{N, 4}\right)$, so that if $\widehat{C}$ is the vertical projection of a circular defining curve for some $G^{\alpha}$, where $\widehat{C}$ is a defining loop for $\widehat{G}$ passing through at least four parabolic fixed points, then there is a $g \in G$, and there is a $j$, $1 \leq j \leq N$, so that $\left(x_{j, 1}, x_{j, 2}, x_{j, 3}, x_{j, 4}\right)$ are four successive parabolic fixed points on $g(\widehat{C})$.

Proof. Choose four successive fixed points $\left(x_{1}, x_{2}, x_{3}, x_{4}\right)$ on $\widehat{C}$. It is clear that we can choose $x_{2}$ to be the fixed point of one of $\widehat{g}_{1}, \ldots, \widehat{g}_{3 p-3}$. Then $x_{1}$ lies in one of the two components stabilized by this $\widehat{g}_{i}$; call it $\Delta_{1}$. As in Proposition 4.1, we note that, modulo the action of $\operatorname{Stab}\left(x_{2}\right)$, there are only finitely many parabolic fixed points on the boundary of $\Delta_{1}$, which can be reached by a path that projects to a simple path. We choose a four-sided fundamental polygon $P_{1}$ for the action of $\operatorname{Stab}\left(\Delta_{1}\right)$ on $\Delta_{1}$, where $x_{2}$ lies on the boundary of $P_{1}$. Using the action of $\operatorname{Stab}\left(x_{2}\right)$, we can find a translate of $\widehat{C}$ so that $x_{1}$ also lies on the boundary of $P_{1}$. There is a natural continuation of the sides of $P_{1}$ into $\Delta_{2}$, and there is a natural unique fundamental polygon $P_{2}$ for $\operatorname{Stab}\left(\Delta_{2}\right)$ with these sides. The content of Lemma 5.1 is that there there are a finite number of translates of $P_{2}$, under $\operatorname{Stab}\left(x_{2}\right)$, so that the endpoint of the intersection of this translate of $\widehat{C}$ with $\Delta_{2}$ lies on the boundary of one of these translates of $P_{2}$; i.e., given that the translate of $\widehat{C}$ passes through $x_{1}$ and $x_{2}$, there are only finitely many possibilities for $x_{3}$. For each of the possible choices for $x_{3}$, we repeat the above argument to conclude that, since we can have only a finite amount of twist about $x_{3}$, there are only finitely many possibilities for $x_{4}$.

\section{Some noded SchotTKy Groups that ARE NOT NEOCLASSiCAL}

Consider the set of noded Riemann surfaces of genus 3, where each of these surfaces has exactly three nodes, all corresponding to dividing loops (this is a complex 3-dimensional family of noded surfaces). In this section, we show that there is no neoclassical Schottky group uniformizing any of these noded surfaces. Then, in the next section, we pick a particular such noded surface, and add three more nodes, so that the resulting noded surface is highly symmetric. We choose a particular noded Schottky group representing this noded surface, and show that this noded Schottky group is sufficiently complicated, and hence not neo-classical. We note that it has been conjectured that every closed Riemann surface can be uniformized by a classical Schottky group; some partial results along these lines are known. The moduli space of Riemann surfaces of genus $p$ has a natural compactification, the Deligne-Mumford compactification, obtained by adding noded Riemann surfaces. Our example here shows that the corresponding conjecture for noded Riemann surfaces does not hold; that is, there are noded Riemann surfaces with no neoclassical Schottky uniformizations.

Assume we are given a Schottky group $G$ and we have some set of defining loops $C_{1}, C_{1}^{\prime}, \ldots, C_{p}, C_{p}^{\prime}$ for $G$. Let $\mathcal{D}$ be the standard fundamental domain for $G$ bounded 


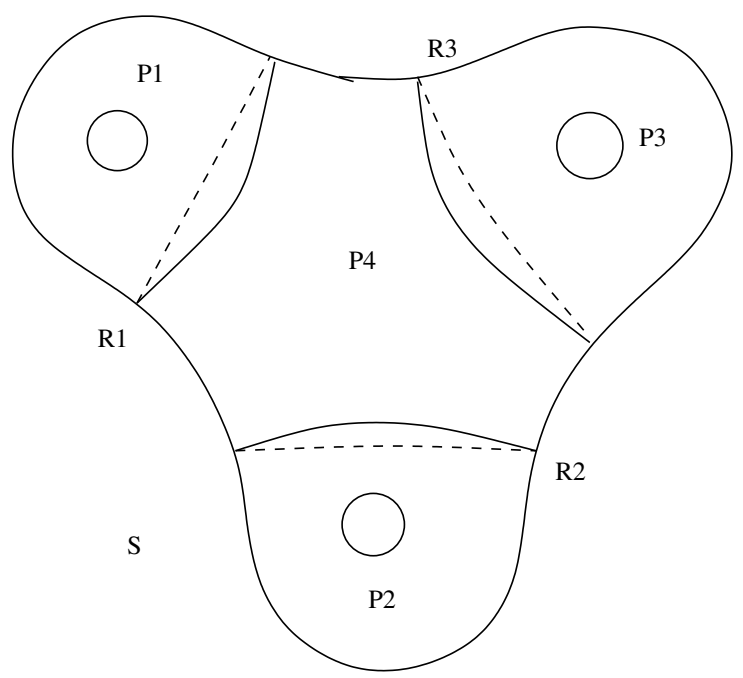

FIGURE 1

by these loops and denote by $P: \Omega(G) \rightarrow S=\Omega(G) / G$ the natural holomorphic covering. Assume that $R \subset S$ is a simple closed curve whose lift to $\mathcal{D}$ consists of pairwise disjoint simple arcs. Each connected component of $P^{-1}(R) \cap \mathcal{D}$ is called a strand of $R$. A strand $\tilde{R}$ of $R$ which has both its endpoints on the same defining loop on the boundary of $\mathcal{D}$ is called essential if it is not homotopically trivial in $\mathcal{D}$ relative to its boundary. Consider a closed Riemann surface $S$ of genus 3 with three homologically trivial, but homotopically distinct and non-trivial, mutually disjoint simple loops, which we think of as red loops. We label these red loops as $R_{1}, R_{2}$ and $R_{3}$. These are chosen so that $S-\left\{R_{1}, R_{2}, R_{3}\right\}$ consists of three one-holed tori, denoted by $P_{1}, P_{2}$ and $P_{3}$, and one 3 -holed sphere, denoted by $P_{4}$. We assume that $R_{i}$ lies on the boundary of $P_{i}, i=1,2,3$ (see Figure 1).

6.1. Generators. Before proceeding, we give an explicit example showing that the noded surface $\widehat{S}$, obtained by shrinking all three red loops, can be uniformized by a noded Schottky group. We remark that it was shown by Hidalgo [3] that every noded Riemann surface can be represented by at least one noded Schottky group. Let $A_{1}, B_{1}, A_{2}, B_{2}, A_{3}, B_{3}$ be a standard homology basis on some surface $S$ of genus 3 as shown in Figure 2 In the same figure are shown the oriented simple loops $R_{1}, R_{2}, R_{3}, W_{1}, W_{2}, W_{3}$. We have that $R_{j}$ is homotopically equivalent to $\left[A_{j}, B_{j}\right]=A_{j} B_{j} A_{j}^{-1} B_{j}^{-1}$, for $j=1,2,3$. We also have that the loops $W_{1}, W_{2}$ and $W_{3}$ are respectively freely homotopic to $B_{3}^{-1} B_{1}^{-1} B_{2}^{-1}, A_{2} A_{1}^{-1}$ and $A_{1} A_{3}^{-1}$. The three loops $W_{1}, W_{2}$ and $W_{3}$ are pairwise disjoint and homologically independent. In particular, they determine a Schottky covering (with corresponding Schottky group $G) P: \Omega(G) \rightarrow S$, defined by the smallest normal subgroup $N \subset \pi_{1}(S)$ containing the elements $w_{1}, w_{2}, w_{3}$, corresponding to the loops $W_{1}, W_{2}, W_{3}$. Let us denote by $d_{1}, d_{2}, d_{3}$ generators of $G$ so that there is a fundamental set of loops for $G$, say $C_{1}, C_{1}^{\prime}, C_{2}, C_{2}^{\prime}, C_{3}, C_{3}^{\prime}$, where $P\left(C_{j}\right)=W_{j}$ and $d_{j}\left(C_{j}\right)=C_{j}^{\prime}$ for $j=1,2,3$. Projecting the elements corresponding to $R_{1}, R_{2}, R_{3}$ to $G=\pi_{1}(S) / N$, we obtain the corresponding elements $r_{1}=d_{1} d_{2} d_{3}^{-1} d_{1}^{-1} d_{3} d_{2}^{-1}, r_{2}=d_{1} d_{2} d_{1}^{-1} d_{2}^{-1}$ 


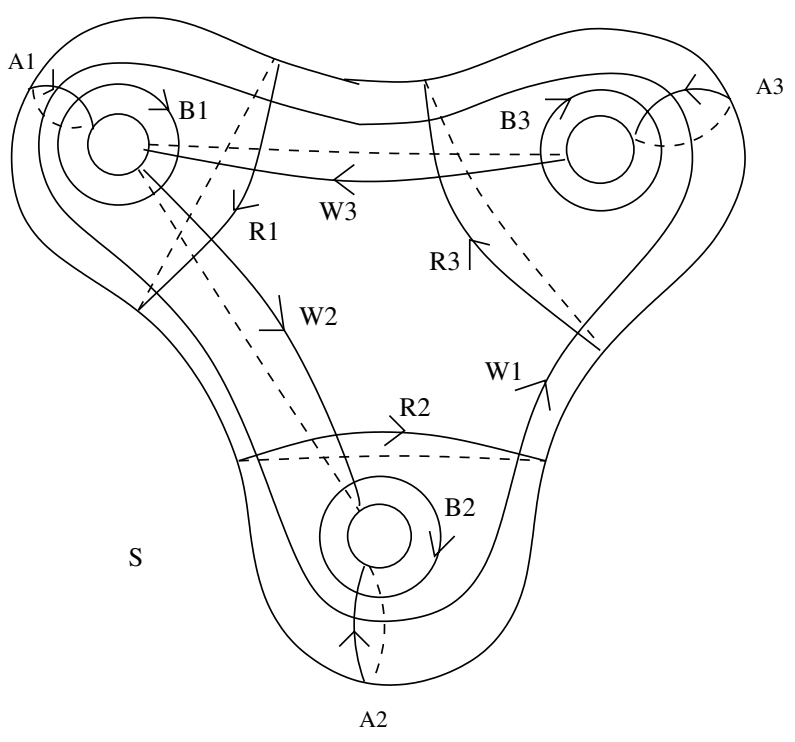

FIGURE 2.

and $r_{3}=d_{1} d_{3}^{-1} d_{1}^{-1} d_{3}$. These three words in the free group $G$ are obviously not powers, and they obviously generate non-conjugate cyclic subgroups. Hence they are pinchable 11 We have shown that $\widehat{S}$ (the noded surface obtained from $S$ after pinching the loops $R_{1}, R_{2}$ and $R_{3}$ ) can be represented by a noded Schottky group.

Theorem 6.1. Let $\widehat{S}$ be some noded Riemann surface of genus 3 formed by pinching the three loops $R_{1}, R_{2}$ and $R_{3}$. Let $\widehat{G}$ be any noded Schottky group so that $\Omega^{+}(\widehat{G}) / \widehat{G}=\widehat{S}$. Then $\widehat{G}$ is not neoclassical.

6.2. The planar graph. We assume that there is a neoclassical noded Schottky group $\widehat{G}$ representing $\widehat{S}$, and we assume that we are given some set of defining loops $\widehat{C}_{1}, \ldots, \widehat{C}_{3}^{\prime}$ for $\widehat{G}$, where the $\widehat{C}_{i}$ are all Euclidean circles. We will not need the explicit generators pairing these. Let $G$ be some Schottky group in a relative conical neighborhood of $\widehat{G}$. Let $C_{1}, \ldots, C_{3}^{\prime}$ be the defining loops for $G$ obtained via vertical projection, and let $R_{1}, R_{2}, R_{3}$ be the geodesics on $S=\Omega(G) / G$ corresponding to the three red loops. Let $\mathcal{D}$ be the fundamental domain for $G$ bounded by $\widehat{C}_{1}, \ldots, \widehat{C}_{3}^{\prime}$. Consider the planar graph $\mathcal{G}$ obtained as follows. We shrink the six defining loops on the boundary of $\mathcal{D}$ to points which become the vertices of $\mathcal{G}$; the edges of $\mathcal{G}$ are the strands of the liftings of $R_{1}, R_{2}$ and $R_{3}$ to $\mathcal{D}$. It is clear that $\mathcal{G}$, as an embedded graph, is well defined up to a homeomorphism of $\widehat{\mathbb{C}}$.

Lemma 6.1. No edge of $\mathcal{G}$ has both endpoints at the same vertex.

Proof. If we had such an edge, starting and ending say at $C_{i}$, then the corresponding defining loop $\widehat{C}_{i}$ would not be simple.

We assume, from here on, that $\mathcal{G}$ contains no edge with both endpoints at the same vertex.

\footnotetext{
${ }^{1}$ This particular example is closely related to some families of examples found by James Blumling and Vinitha Jacob, two high school students who worked with the second author.
} 
Lemma 6.2. Given any two vertices of $\mathcal{G}$, there is at most one edge connecting them.

Proof. Suppose there were two edges starting at $C$ and ending at $C^{\prime}$, where $C$ and $C^{\prime}$ are each one of loops $C_{1}, \ldots, C_{3}^{\prime}$. Then, in $\Omega(G)$, there is an arc of the corresponding defining loop $C$ between these two edges, and there is likewise an arc of the corresponding defining loop $C^{\prime}$. When we contract the red edges to points, these two arcs become arcs of the corresponding defining circles $\widehat{C}$ and $\widehat{C}^{\prime}$, which are now required to pass through the same two parabolic fixed points; this cannot be.

We assume, from here on, that $\mathcal{G}$ is such that there is at most one edge connecting any two vertices. For each vertex $C_{i}$ (respectively, $C_{i}^{\prime}$ ), we denote the number of edges of $\mathcal{G}$ ending at $C_{i}$ (respectively, $C_{i}^{\prime}$ ) by $N_{i}$ (respectively, $N_{i}^{\prime}$ ). Since $C_{i}$ and $C_{i}^{\prime}$ are identified by an element of $G, N_{i}=N_{i}^{\prime}$. Since $N_{i}=N_{i}^{\prime}, \sum N_{i}$ is the total number of edges of $\mathcal{G}$. For $i=1,2,3$, let $V_{i}$ denote the projection of (the loop) $C_{i}$ to $S$. Observe that $V_{1}, V_{2}, V_{3}$ are three homologically independent simple disjoint loops on $S$. Then $N_{i}$ is the total number of points of intersection of $V_{i}$ with the dividing loops $R_{1}, R_{2}$ and $R_{3}$. Since the $R_{j}$ are dividing loops, each $N_{i}$ is even. Similarly, let $M_{j}$ denote the sum of the number of crossings of $R_{j}$ with $V_{1}, V_{2}$ and $V_{3}$. Again, $M_{j}$ is necessarily even. It is clear that $M_{j}$ is the number of strands of liftings of $R_{j}$; hence $\sum M_{j}=\sum N_{i}$ is the total number of edges of $\mathcal{G}$.

Proposition 6.1. For $j=1,2,3, M_{j}>2$.

Proof. We cannot have $M_{j}=0$, for then the loop $R_{j}$ does not cross any of the $V_{i}$, so it lifts to a loop, which cannot be. If $M_{j}=2$, then $R_{j}$ crosses exactly one of the $V_{i}$, say it is $V_{1}$, and it crosses $V_{1}$ twice. Hence the lift of $R_{j}$ defines exactly two essential strands. Both of these strands have one endpoint on $C_{1}$ and the other endpoint on $C_{1}^{\prime}$, which also cannot be.

Since each $M_{j} \geq 4$, the total number of strands is at least 12 . However, since we do not have more than one edge from any one vertex to any other vertex, and each $N_{i}$ is even, we have that the total number of strands, which is the same as the total number of edges, is at most 12 . Hence the number of edges is exactly 12 , and there are exactly four edges emanating from each vertex. It is easy to see that, up to isomorphism, there is exactly one graph with 6 vertices, 4 edges at each vertex, no edge having the same vertex at both ends, and no two edges having the same pair of vertices at their ends. We note that this graph is homogeneous, and that it has essentially only one embedding in the extended complex plane. We can think of the vertices as the points $0, \infty, \pm 1$ and $\pm i$, and the edges as lying on the real and imaginary axes, and on the unit circle. Observe that this graph has as a group of isometries the symmetric group on four letters, $\mathcal{S}_{4}$, generated by the Möbius transformations $A(z)=i z$ and $B(z)=\frac{1-z}{1+z}$. We may think of the vertices as the midpoints of the faces of a cube; that is, $\mathcal{G}$ is the dual graph to the graph on $\mathbb{S}^{2}$ obtained from the regular cube by projection from the origin. We have shown that the strands of $\mathcal{G}$ divide $D$ into 8 regions, corresponding to the vertices of the cube. We have the natural projection from $D$ onto $S$, which is divided into the 4 subsurfaces $P_{1}, P_{2}, P_{3}$ and $P_{4}$ by the red loops. Hence we can identify each of the corresponding 8 regions of $\mathcal{D}$ as projecting onto one of these four subsurfaces. Further, for every strand of $\mathcal{G}$, exactly one of the two sides of this strand projects 


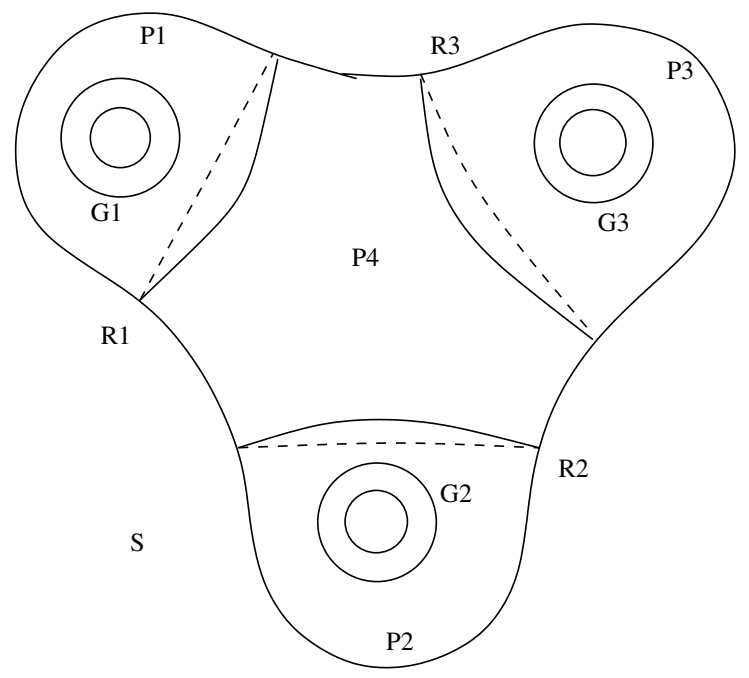

FIGURE 3.

onto the 3-holed sphere, $P_{4}$, and the other side projects onto one of the surfaces $P_{1}, P_{2}$ or $P_{3}$. It follows that 4 of these regions project onto $P_{4}$, and that, for each of these regions projecting onto $P_{4}$, we must have that each of the 3 neighboring regions projects onto a distinct $P_{j}, j=1,2,3$. It is easy to see that this is not possible (try to put a value 1, 2, 3 or 4 to each vertex of the cube so that (i) for any two adjacent vertices, one of the values is 4 , and (ii) at each vertex labeled 4 , all three values 1, 2 and 3 occur at an adjacent vertex). This completes the proof of the fact that there is no neoclassical noded Schottky group representing any of our noded Riemann surfaces.

\section{A SUfficiently COMPlicated NOded SCHOTtKy GROuP}

In this section, we construct a particular example of a sufficiently complicated maximally noded Schottky group of genus 3. The construction starts with the surface $S_{0}$ of the last section, with the three red loops $R_{1}, R_{2}$ and $R_{3}$ on it. We enlarge this set of simple disjoint loops by adjoining three non-dividing loops, which we think of as green loops, $G_{1}, G_{2}$ and $G_{3}$, where, for $i=1,2,3, G_{i}$ lies in the subsurface $P_{i}$ (see Figure 3 ).

It was shown by Hidalgo 3 that every noded Riemann surface can be uniformized by at least one noded Schottky group. However, for our purposes, we will need to carefully choose our noded Schottky group.

7.1. Explicit conformal specifications. We make some additional requirements on the explicit conformal structure on $S_{0}$; we require that $S_{0}$ admits a conformal automorphism $\phi$ of degree 3 , where $\phi\left(R_{1}\right)=R_{2}, \phi\left(R_{2}\right)=R_{3}, \phi\left(R_{3}\right)=R_{1}, \phi\left(G_{1}\right)=$ $G_{2}, \phi\left(G_{2}\right)=G_{3}$ and $\phi\left(G_{3}\right)=G_{1}$. We also require that $S_{0}$ admits a reflection, $\psi$, with the properties that the three dividing geodesics $R_{1}, R_{2}, R_{3}$ are invariant but not pointwise fixed under $\psi$, and the three non-dividing geodesics $G_{1}, G_{2}, G_{3}$ are pointwise fixed under $\psi$. There is a two real parameter family of such surfaces; we choose $S_{0}$ to be any surface within this family. 


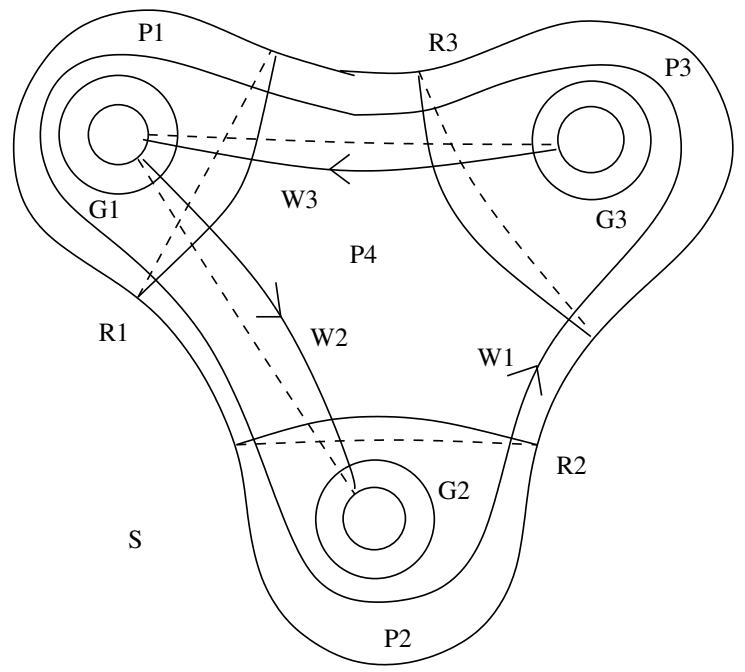

FIGURE 4.

7.2. Explicit topological specifications. We next need to choose a particular set of loops to define a Schottky group. We choose the loops $W_{1}, W_{2}, W_{3}$ to have the following properties (see Figure 4).

The loop $W_{1}$ crosses each of $R_{1}, R_{2}$ and $R_{3}$ exactly twice, is disjoint from each of $G_{1}, G_{2}$ and $G_{3}$, is invariant under the rotation $\phi$, and is pointwise fixed by the reflection $\psi$. The loop $W_{2}$ crosses each of $R_{1}$ and $R_{2}$ exactly twice, is disjoint from $R_{3}$, crosses each of $G_{1}$ and $G_{2}$ exactly once, is disjoint from $G_{3}$, and is invariant under the reflection, $\psi$. The loop $W_{3}=\phi^{-1}\left(W_{2}\right)$. One easily sees that $\phi\left(W_{2}\right)$ is freely homotopic to the product $W_{3}^{-1} \cdot W_{2}^{-1}$, from which it follows that the conformal map $\phi$ lifts to the Schottky group $G_{0}$ defined by these three loops. Since $\psi$ preserves each of $W_{1}, W_{2}, W_{3}$, it also lifts to an action on $\Omega\left(G_{0}\right)$. It follows from well-known properties of Schottky groups that the lift $\tilde{\phi}$ of $\phi$ is a Möbius transformation, and that the lift $\tilde{\psi}$ of $\psi$ is an orientation-reversing Möbius transformation with a Euclidean circle of fixed points.

Proposition 7.1. For the Schottky group $G_{0}$, the set of loops $R_{1}, R_{2}, R_{3}, G_{1}, G_{2}, G_{3}$ is pinchable.

Proof. We need to show that none of these loops lifts to a loop, and that no two of them define conjugate elements of $G_{0}$. Since $G_{1}$ crosses $W_{2}$ exactly once, it does not lift to a loop; likewise $G_{2}$ and $G_{3}$ do not lift to loops. If say $R_{2}$ were to lift to a loop, then by cutting and pasting with $W_{2}$, we would obtain a loop that lifts to a loop and that crosses $W_{1}$ exactly once, which cannot be. Hence $R_{1}, R_{2}$ and $R_{3}$ do not lift to loops. If two of these loops were to define the same element of $G_{0}$, then they would have the same crossing pattern with the triple of loops $W_{1}, W_{2}, W_{3}$. But in fact, if one looks at the $6 \times 3$ matrix of geometric crossings of these loops with the $W_{i}$, no two columns are the same; hence no pair of these loops can define the same or conjugate elements of $G_{0}$.

We have shown that the loops $R_{1}, R_{2}, R_{3}, G_{1}, G_{2}, G_{3}$ are pinchable; hence we can define the noded Schottky group $\widehat{G}_{0}$ obtained by pinching these loops. We require 
that the pinching be done so that the maps $\tilde{\phi}$ and $\tilde{\psi}$ are defined and normalize the Schottky group at each step of the pinching; hence they are defined and normalize $\widehat{G}_{0}$. On the noded Riemann surface $\widehat{S}_{0}$, obtained by pinching these six loops, the loops $\widehat{W}_{1}, \widehat{W}_{2}$ and $\widehat{W}_{3}$ are well defined. We remark that, as a consequence of the fact that $\tilde{\psi}$ lifts to a homeomorphism of $\Omega^{+}\left(\widehat{G}_{0}\right)$, we have that the lift of $\tilde{\psi}$ is a fractional linear reflection, from which it follows that its fixed point set is a Euclidean circle. Since $\widehat{W}_{1}$ is pointwise fixed under $\tilde{\psi}$, it follows that the projection of this circle of fixed points, including the parabolic fixed points it passes through, is exactly $\widehat{W}_{1}$.

7.3. It is easy to observe that the loops $W_{2}$ and $W_{3}$ are parallel going from $R_{1}$ to $G_{1}$ and back to $R_{1}$; hence there are lifts of $\widehat{W}_{2}$ and $\widehat{W}_{3}$ that are parallel between three successive parabolic fixed points. We need to check that this same phenomenon occurs for every choice of defining loops. In what follows, we assume that we are given some set of three defining loops $B_{1}, B_{2}$ and $B_{3}$ for our particular Schottky group, which we refer to as black loops. We assume that it does not happen that there are two arcs of black loops that are parallel between three successive crossings of red and green loops. In what follows, we denote the homology intersection number of the loops, $A$ and $B$ by $A \times B$, and we denote the geometric intersection number by $A \bullet B$.

Proposition 7.2. Let $\tilde{S} \rightarrow S$ be a regular covering of a surface, where $\tilde{S}$ is planar, and let $A$ and $B$ be loops on $S$ that lift to loops on $\tilde{S}$. Then $A \times B=0$.

Proof. We can assume without loss of generality that $A$ and $B$ cross a finite number of times. Let $\tilde{A}$ be any lift of $A$, and let $\tilde{B}_{1}, \ldots, \tilde{B}_{k}$ be the set of lifts of $B$ that $\operatorname{cross} \tilde{A}$. Since $\tilde{S}$ is planar, for each $i, \tilde{A} \times \tilde{B}_{i}=0$. The result follows from the fact that $A \times B=\sum \tilde{A} \times \tilde{B}_{i}$.

Corollary 7.1. If $B$ is a black loop, then $\sum_{i=1}^{3} B \times G_{i}=0$.

Proof. Observe that, after appropriate orientation, $W_{1}$ is homologous to $G_{1}+G_{2}+$ $G_{3}$. Since $B$ and $W_{1}$ both lift to loops, $\sum B \times G_{i}=B \times W_{1}=0$.

Proposition 7.3. No $B_{j}$ lies entirely within any one of the $P_{i}$, i.e., every black loop crosses at least one red loop.

Proof. It is essentially obvious that no $B_{i}$ can lie entirely within the planar region $P_{4}$, for every simple homotopically non-trivial loop in $P_{4}$ is parallel to the boundary, i.e., it is freely homotopic to a red loop. If some $B_{j}$ were to lie in one of the tori $P_{1}$, $P_{2}$ or $P_{3}$, then the commutator of this loop with a conjugate loop would be freely homotopic to the red loop on the boundary, which cannot be, as the red loop does not lift to a loop.

In what follows, the subsurfaces $P_{1}, P_{2}, P_{3}$ are called the tori, and the subsurface $P_{4}$ is called the planar region. Also, the arcs of black loops in each of the tori are called strands. The proposition above shows that each black loop contains at least one strand. For some purposes, we will replace the red boundary of a torus by a point, so that we can think of the torus as closed. If two strands cross at this point, then the endpoints of one of them on the red boundary separates the endpoints of the other. In this case, we say that the strands cross on the red boundary. Otherwise, we say that they are parallel. Of course, two simple homotopically nontrivial loops on a torus that meet at exactly one point either cross at that point or are homotopic (up to orientation). 
Proposition 7.4. Every $R_{i}$ is crossed by at least one $B_{j}$, and every $G_{i}$ is crossed by at least one $B_{j}$.

Proof. Every loop that is not crossed by some $B_{j}$ lifts to a loop, and we require that the $R_{i}$ and the $G_{i}$ do not lift to loops.

Proposition 7.5. Every black loop contains at least two strands.

Proof. We know from the above that every black loop contains at least one strand. Suppose for example that $B_{1}$ crosses only $R_{1}$ at exactly two points. Then $B_{1}$ consists of exactly a strand in $P_{1}$ and an arc in the planar region. Since the arc in $P_{4}$ is homotopically non-trivial and not parallel to the boundary of $P_{4}$, it separates $P_{2}$ from $P_{3}$. It follows that $\phi\left(B_{1}\right)$ crosses $B_{1}$ in $P_{4}$, and only in $P_{4}$. Appropriately cutting and pasting these two loops, we obtain a new simple homotopically nontrivial loop that also lifts to a loop, and that lies entirely in $P_{4}$, which cannot be.

Proposition 7.6. Every torus contains at least two strands.

Proof. Since each $G_{i}$ is crossed by at least one black loop, each torus contains at least one strand. If say $P_{1}$ were to contain only one strand, then we could find a simple homotopically non-trivial loop in $P_{1}$ that does not cross that strand, and hence does not cross any black loop. Such a loop necessarily lifts to a loop, and its commutator with a conjugate loop also lifts to a loop. But this commutator is freely homotopic to $R_{1}$, which does not lift to a loop.

Proposition 7.7. If $A_{1} \neq A_{2}$ are distinct strands in the torus $P_{i}$, where $A_{1} \bullet G_{i} \neq$ $A_{2} \bullet G_{i}$, then $A_{1}$ and $A_{2}$ cross on $R_{i}$.

Proof. This is immediate from the fact that, on the closed torus, $A_{1}$ and $A_{2}$ are homotopically non-trivial and distinct.

Proposition 7.8. If there are exactly two strands $A_{1}$ and $A_{2}$ in some $P_{i}$, then $A_{1} \bullet G_{i} \neq A_{2} \bullet G_{i}$. Further, either both $A_{1}$ and $A_{2}$ cross $G_{i}$, or, after appropriate labeling, $A_{1} \bullet G_{i}=1$ and $A_{2} \bullet G_{i}=0$.

Proof. Since $G_{i}$ does not lift to a loop, one of the strands in $P_{i}$ must cross $G_{i}$; we can assume that it is $A_{1}$. If $A_{2}$ also crosses $G_{i}$, then we cannot have that $A_{1} \bullet G_{i}=A_{2} \bullet G_{i}$, for then, after perhaps reorienting one of them, we would have that $A_{1}$ and $A_{2}$ are parallel through a red loop, at least one green loop, and then a red loop. One also easily sees that if $A_{1} \bullet G_{i}>1$, then $A_{2} \bullet G_{i} \neq 0$.

Proposition 7.9. Let $A$ be a strand in the torus $P_{i}$. Then $A \bullet G_{i} \leq 3$.

Proof. If $A \bullet G_{i} \geq 4$, then there would be two distinct lifts of $A$, starting at some lift of $G_{i}$, that would be parallel between two successive lifts of $G_{i}$, followed by a lift of $R_{i}$, contradicting our basic assumption that $\widehat{G}_{0}$ is not sufficiently complicated.

Proposition 7.10. There are at most three strands inside any torus. Further, if there are three strands in some $P_{i}$, then two of them are parallel without crossing $G_{i}$, and the third crosses $G_{i}$ exactly once.

Proof. Suppose there are $K>1$ strands $A_{1}, \ldots, A_{K}$ in $P_{i}$, where, for $j=1, \ldots, K-$ $1, A_{j} \bullet G_{i} \geq A_{j+1} \bullet G_{i}$. We have already seen that $A_{1} \bullet G_{i}>0$. We cannot have two parallel strands crossing $G_{i}$ the same positive number of times, hence 
$A_{j} \bullet G_{i}>A_{j+1} \bullet G_{i}$, unless $A_{j} \bullet G_{i}=0$. In particular, $A_{2} \bullet G_{i}<A_{1} \bullet G_{i}$. We first assume that $A_{2} \bullet G_{i}>0$, then $A_{1} \bullet G_{i}>1$, from which it follows that every strand in $P_{i}$ crosses $G_{i}$. Continuing with the assumption that $A_{2} \bullet G_{i}>0$, we next observe that we cannot have that $A_{1} \bullet G_{i}=3$, and $A_{2} \bullet G_{i}=2$. For lifts of $A_{1}$ and $A_{2}$ would then be parallel through two successive lifts of $G_{i}$, followed by a lift of $R_{i}$. We conclude that if $A_{2} \bullet G_{i}>0$, then $K=2, A_{2} \bullet G_{i}=1$, and either $A_{1} \bullet G_{i}=3$ or $A_{1} \bullet G_{i}=2$. We next take up the case that $A_{2} \bullet G_{i}=0$. Then, since the lift of $G_{i}$ must be primitive, $A_{1} \bullet G_{i}=1$. If $K \geq 3$, then none of the strands $A_{2}, \ldots, A_{K}$ cross $G_{i}$, and they are all parallel. Since any pair of these parallel strands must come from distinct red loops other than $R_{i}$, and must go to distinct red loops, there can be at most two of these strands. We have shown that $K \leq 3$.

Proposition 7.11. At least one of the $P_{i}$ has three strands in it.

Proof. If not, then all three of the tori would contain exactly two strands. Since each black loop must contain at least two strands, it follows that each of the black loops contains exactly two strands. Then each of the two ends of one of the strands from $P_{1}$ connect to each of the two ends of one of the strands of $P_{2}$, and each of the two ends of the other strand in $P_{1}$ connects to each of the two ends of one of the strands of $P_{3}$. Since the endpoints of the first strand separate the endpoints of the second strand on $R_{i}$, these two black loops must intersect in $P_{4}$, which cannot be.

We assume, from here on, that there are three strands, $A_{1}, A_{2}, A_{3}$, of black loops in $P_{1}$, where $A_{1} \bullet G_{1}=1$, and $A_{2}$ and $A_{3}$ are parallel with $A_{2} \bullet G_{1}=A_{3} \bullet G_{1}=0$.

Proposition 7.12. Of the six endpoints of strands on $R_{1}$, three connect to $R_{2}$ and three connect to $R_{3}$. Also, one end of $A_{1}$ connects with $R_{2}$ and the other end connects with $R_{3}$.

Proof. We orient $A_{1}, A_{2}$ and $A_{3}$ so that $A_{2}$ and $A_{3}$ are parallel as oriented curves. Then $A_{2}$ and $A_{3}$ cannot both come from the same red loop other than $R_{1}$, and they cannot both go to the same red loop other than $R_{1}$. Hence we can assume that $A_{2}$ comes from $R_{2}$ and that $A_{3}$ comes from $R_{3}$. Then either $A_{2}$ also goes to $R_{2}$ and $A_{3}$ also goes to $R_{3}$, or $A_{2}$ goes to $R_{3}$ and $A_{3}$ goes to $R_{2}$. In either case, since $A_{1}$ crosses $A_{2}$ and $A_{3}$ on $R_{1}$, both endpoints of $A_{1}$ cannot go to $R_{2}$, and both endpoints of $A_{1}$ cannot go to $R_{3}$. Hence one end of $A_{1}$ connects with $R_{2}$ and one end connects with $R_{3}$.

Proposition 7.13. $P_{2}$ and $P_{3}$ each contain exactly two strands.

Proof. Suppose there were three strands in $P_{2}$, call them $A_{1}^{\prime}, A_{2}^{\prime}$ and $A_{3}^{\prime}$, labelled as above, so that $A_{1}^{\prime} \bullet G_{2}=1$ and $A_{2}^{\prime} \bullet G_{2}=A_{3}^{\prime} \bullet G_{3}=0$, and oriented as above, so that $A_{2}^{\prime}$ and $A_{3}^{\prime}$ are parallel as oriented curves. Then three of the endpoints of strands in $P_{1}$ connect with three of the strands in $P_{3}$, and three of the endpoints of strands of $P_{2}$ connect with strands of $P_{3}$. This implies incidentally that $P_{3}$ also contains three strands. In any case, $A_{1}$ is the middle strand from $P_{1}$ connecting to $P_{2}$, and $A_{1}^{\prime}$ is the middle strand from $P_{2}$ connecting to $P_{1}$. We have shown that there is exactly one black loop, call it $B_{1}$, containing the strands $A_{1}$ and $A_{1}^{\prime}$. We now have that $B_{1} \bullet G_{1}=B_{1} \bullet G_{2}=1$, and that both $B_{2}$ and $B_{3}$ do not cross either $G_{1}$ or $G_{2}$. This implies that $G_{1}$ and $G_{2}$ define conjugate elements of the Schottky group $G$, which they do not. 
We now observe that we have reached our final contradiction. For we have shown that, of the three black loops, one of them crosses $G_{1}$ and has three strands; the second does not cross $G_{1}$ and has two strands, the second strand lying in $P_{2}$, while the third black loop has two strands, one lying in $P_{1}$ and not crossing $G_{1}$, while the other lies in $P_{3}$. The second black loop cannot cross $G_{2}$, for that would contradict Corollary 7.1 likewise, the third black loop cannot cross $G_{3}$. That leaves us with the first black loop crossing each of $G_{1}, G_{2}$ and $G_{3}$, and crossing each of them once, which also contradicts Corollary 7.1 .

\section{REFERENCES}

[1] V. Chuckrow. On Schottky groups with applications to Kleinian groups. Annals of Math., 88:47-61, 1968. MR.0227403 (37:2987)

[2] L. Gerritzen and F. Herrlich. The extended Schottky space. J. Reine Angew. Math., 389:190208, 1988. MR0953671 (89h:32043)

[3] R.A. Hidalgo. The noded Schottky space. London Math. Soc., 73:385-403, 1996. MR.1397694 (97h:32031)

[4] R.A. Hidalgo. Noded Fuchsian groups. Complex Variables, 36:45-66, 1998. MR1637340 (99c:30074)

[5] T. Jørgensen and A. Marden. Algebraic and geometric convergence of Kleinian groups. Math. Scand., 66:47-72, 1990. MR1060898 (91f:30068)

[6] T. Jørgensen, A. Marden, and B. Maskit. The boundary of classical Schottky space. Duke Math. J., 46:441-446, 1979. MR0534060(80k:32028)

[7] L. Keen, B. Maskit, and C. Series. Geometric finiteness and uniqueness for kleinian groups with circle packing limit sets. J. Reine Angew. Math., 436:209-219, 1993. MR.1207287 (94b:30053)

[8] I. Kra and B. Maskit. Pinched two component Kleinian groups. In Analysis and Topology, pages 425-465. World Scientific Press, 1998. MR1667825 (99m:20119)

[9] A. Marden. Schottky groups and circles. In Contributions to Analysis, pp. 273-278. Academic Press, New York and London, 1974. MR0361058 (50:13504)

[10] B. Maskit. A characterization of Schottky groups. J. d'Analyse Math., 19:227-230, 1967. MR0220929 (36:3981)

[11] B. Maskit. On free Kleinian groups. Duke Math. J., 48:755-765, 1981. MR0782575 (86d:30073)

[12] B. Maskit. Parabolic elements in Kleinian groups. Annals of Math., 117:659-668, 1983. MR0701259 (85a:30073)

[13] B. Maskit. Kleinian Groups. Springer-Verlag, Berlin, Heidelberg, New York, 1988. MR0959135 (90a:30132)

[14] B. Maskit. On Klein's combination theorem IV. Trans. Amer. Math. Soc., 336:265-294, 1993. MR1137258 (93e:30088)

[15] B. Maskit. On spaces of classical Schottky groups. Contemporary Math., 256:227-237, 2000. MR:1759682(2001f:30052)

[16] H. Sato. Introduction of new coordinates to Schottky space - the general case. J. Math. Soc. Japan, 35:23-35, 1983. MR0679071 (85a:32031)

[17] Hiro-o Yamamoto. Squeezing deformations in Schottky spaces. J. Math. Soc. Japan, 31:227243, 1979. MR0527540 (80g:30029)

[18] Hiro-o Yamamoto. An example of a non-classical Schottky group. Duke Math. J., 63:193-197, 1991. MR1106942 (92m:30078)

Departamento de Matemática, Universidad Tecnica Federico Santa Maria, ValpaRAÍso, CHILE

E-mail address: ruben.hidalgo@usm.cl

Department of Mathematics, SUny at Stony Brook, Stony Brook, New York 117943651

E-mail address: bernie@math.sunysb.edu 